\begin{tabular}{|c|l|}
\hline Title & Tropical Cyclone Tracking U sing a Neighbor Enclosed A rea Tracking A I gorithm \\
\hline Author(s) & Satake, Y uya; Inatsu, Masaru; Mori, Masato; Hasegawa, A kira \\
\hline Citation & $\begin{array}{l}\text { Monthly Weather Review, 141(10), 3539-3555 } \\
\text { https://doi.org/10.1175MWR-D-12-00092.1 }\end{array}$ \\
\hline Issue Date & 2013-10 \\
\hline Doc URL & http://hdl.handle.net/2115/55290 \\
\hline Rights & ○ Copyright 2013 A merican Meteorological Society (A MS). \\
\hline Type & article \\
\hline File Information & mwr-d-12-00092.1.pdf \\
\hline
\end{tabular}

Instructions for use 


\title{
Tropical Cyclone Tracking Using a Neighbor Enclosed Area Tracking Algorithm
}

\author{
YUYA SATAKE \\ Tokio Marine and Nichido Risk Consulting Co. Ltd., Tokyo, Japan \\ MASARU INATSU \\ Graduate School of Science, Hokkaido University, Sapporo, Japan \\ MASATO MORI \\ Atmosphere and Ocean Research Institute, The University of Tokyo, Kashiwa, Chiba, Japan \\ AKIRA HASEgAwA \\ International Center for Water Hazard and Risk Management, Public Works Research Institute, Tsukuba, Ibaraki, Japan
}

(Manuscript received 29 March 2012, in final form 3 April 2013)

\begin{abstract}
Tropical cyclone (TC) tracking is essential for calculating TC statistics from gridded datasets. A new method for TC tracking is presented here using neighbor enclosed area tracking (NEAT), which is based on the temporal overlap of enclosed areas above a vorticity threshold and differs from the widely used neighbor point tracking (NPT) method. The parameters of cyclone intensity, vertical-shear, and warm-core criteria were intensively tuned for NEAT and NPT. When these criteria were optimized for the typhoon tracks observed in the western North Pacific based on the Japanese 25-yr Reanalysis Project (JRA-25)/Japan Meteorological Agency (JMA) Climate Data Assimilation System (JCDAS) dataset, the NEAT and NPT algorithms captured approximately $85 \%$ of typhoons with little qualitative distortion in the spatial distribution and temporal variability of the TC track density. The grid system dependency of the algorithms was tested by applying NEAT and NPT to a high-resolution general circulation model output. The method presented here can also provide realistic statistics on the TC size, the extratropical transition timing, and the meridional heat transport.
\end{abstract}

\section{Introduction}

Tropical cyclone (TC) tracking is an indispensable data processing tool for calculating TC statistics from model output. A widely used method for TC tracking is neighbor point tracking (NPT), in which cyclone centers are identified as local extrema of a climatic variable such as the relative vorticity and a cyclone track is derived by temporally connecting the cyclone centers. The NPT technique is common in TC tracking (see the references below) and extratropical cyclone tracking (Ulbrich et al. 2009). To exclude extratropical cyclones, a TC identification algorithm is typically implemented in the tracking

Corresponding author address: Dr. Masaru Inatsu, Graduate School of Science, Hokkaido University, Rigaku bldg. 8, N10W8, Kita, Sapporo 0600810, Japan.

E-mail: inaz@mail.sci.hokudai.ac.jp code itself; alternatively, the algorithm can be conducted in a post-tracking process. Early in the development of TC simulations, Broccoli and Manabe (1990) performed TC identification by restricting the location of the cyclone center. This method is still used for TC identification (McDonald et al. 2005). As an alternative to the location condition, Haarsma et al. (1993) used the warm-core structure in midtropospheric temperature anomalies. The parameters were determined arbitrarily, however, because coarse-resolution general circulation models (GCMs) alone produced weak tropical depression systems. Bengtsson et al. (1995) successfully produced TC statistics by using a GCM at a T106 $(\sim 110 \mathrm{~km})$ resolution and using more realistic measures for TC tracking, including several conditions of intensity (relative vorticity, mean sea level pressure, and maximum surface wind speed), vertical wind shear, warm-core structure, and duration. Subsequent TC tracking studies 
based on GCM output followed this method (Hasegawa and Emori 2005; Oouchi et al. 2006; Murakami and Wang 2010; Sugi et al. 2002). Bengtsson et al. (2007) adopted simpler conditions, such as an intensity index, a warmcore criterion, and a duration condition. As stated above, all TC tracking methods include some parameters that should be adjusted to the data resolution; of course, the resolution dependence problem could be mostly resolved by using a uniform resolution. Walsh et al. (2007) noted the diversity of setting parameters in TC identification from GCM data and found that the wind speed criterion should vary approximately linearly with resolution. This estimate is helpful for parameter tuning when comparing GCMs with different resolutions. Recent higher-resolution model simulations have demonstrated the ability to capture realistic TC statistics (e.g., Knutson et al. 2007; Manganello et al. 2012; Murakami and Wang 2010; Oouchi et al. 2006). Hence, in future studies, parameters used for TC identification in model simulations should ideally be the same as those in spatially dense data, such as numerical weather forecasts. Apart from this resolutiondependence problem, given two identical datasets with different grid systems, the NPT method will produce different results unless the datasets are spatially interpolated or aggregated into a common grid system in a pretracking process (Bengtsson et al. 2007; Manganello et al. 2012). Although this additional process is necessary in the case of NPT because of its dependence on the grid system, aggregation or interpolation can indeed be effective for mitigating this problem in NPT algorithms. In this paper, the resolution-dependence problem in TC simulations for different-resolution models is distinguished from the dependence on the grid system.

TCs are also characterized by their size, their extratropical transition timing (if any), and their meridional heat transport. The size of TCs has been approximately measured from observation data in a number of studies (Kimball and Mulekar 2004; Lee et al. 2010; Merrill 1984). Sinclair (1997) and Rudeva and Gulev (2007) developed a method for finding the zero contours in the downgradient direction from the cyclone center and applied this technique to extratropical cyclones in the Southern Hemisphere. Furthermore, the extratropical transition occurs when a TC changes into a baroclinic asymmetric structure under a strong vertical-shear environment, and the cyclone often redevelops in the extratropics; many attempts have been made to estimate this transition objectively [see Jones et al. (2003) for a review]. Hart (2003) focused on the front and thermalcore structure of cyclones and proposed that the life cycle can be mapped onto the phase space spanned by these two measures. Evans and Hart (2003) demonstrated that the phase-space method is useful for a further life cycle classification of observed cyclones. Kitabatake (2011) applied the cyclone phase-space technique developed by Hart (2003) to typhoons in the western North Pacific. However, the meridional heat transport of TCs is largely unexplored.

Inatsu (2009) recently proposed an alternative cyclone tracking method called neighbor enclosed area tracking (NEAT), in which enclosed areas that satisfy cyclone conditions are identified. The enclosed cyclone area is then tracked when it overlaps with another area in the subsequent time frame [see Inatsu (2009) and Inatsu and Amada (2013) for more details of the NEAT algorithm]. The NEAT algorithm has three main properties, all of which can be realized in an NPT algorithm. The NEAT algorithm requires few tunable parameters because it is based on a unified method for cyclone identification and cyclone tracking. Additionally, because this method uses finite-area identification rather than local-extremum identification, in principle, the dependence on the data grid system is relieved under the condition that the data are sufficiently dense to resolve the target phenomenon. State-of-the-art NPT algorithms use an interpolation technique to relocalize the extremum between data grid points and prepare a common grid for the comparison of data with different grid systems. Even for temporal connections in NEAT, the overlap method relieves grid-system dependency. The overlap problem for TC tracking is thoroughly discussed in appendix A. In addition, the NEAT algorithm can produce useful quantitative data for cyclone merger and splitting events, the size and shape of eddies, and material and dynamical transport by eddies, whereas these data would be estimated by the NPT algorithm. Accordingly, TC tracking using the NEAT algorithm is an alternative method for extracting TC tracks from reanalysis datasets or from the outputs of a model simulation.

The purpose of the present study is to propose an alternative choice of a TC tracking method using the NEAT algorithm. The new algorithm was applied to a reanalysis dataset with embedded bogus typhoon data. Some parameters of the NEAT algorithm were tuned by comparing the reanalysis data and the typhoon best tracks, both of which were retrieved from the archives of the Japan Meteorological Agency (JMA). The JMA definition of a typhoon is based on a 10-min-average surface wind speed in excess of $17.2 \mathrm{~m} \mathrm{~s}^{-1}$. This study focuses on TCs only in the western North Pacific, although our technique can be applied to TCs anywhere. Section 2 describes the datasets used in this study, section 3 explains tracking methods, and section 4 develops an optimal parameter set for the NEAT and NPT methods for identifying TCs. Section 5 presents the performance of both methods using the optimal parameter set to 
capture the spatial distribution and temporal variations in TC activity in the western North Pacific. Section 6 briefly discusses the grid-system dependence by using high-resolution GCM output. Section 7 addresses other tests that the new method can be applied to, and section 8 provides concluding remarks.

\section{Data}

The data used in this paper are from the Japanese 25-yr Reanalysis Project (JRA-25)/JMA Climate Data Assimilation System (JCDAS) gridded data, for which the grid mesh is $1.25^{\circ} \times 1.25^{\circ}$ and the temporal interval is $6 \mathrm{~h}$ (Onogi et al. 2007). The TCs were extracted over the domain $0^{\circ}-60^{\circ} \mathrm{N}$ and $100^{\circ} \mathrm{E}-180^{\circ}$ from January 1979 to December 2009. Compared with other reanalysis datasets, JRA-25/JCDAS is valuable for setting up a TC tracking algorithm because bogus typhoon data were embedded throughout the period in the dataset (Onogi et al. 2007). Therefore, TCs and their surrounding environment are better reproduced than in other reanalysis datasets (Hatsushika et al. 2006). Applying a tracking algorithm to the dataset provides TC tracks from the reanalysis data. A set of parameters is tuned such that the algorithm detects as many typhoons embedded in the reanalysis data as possible with minimal processing error. The reference is the best-track data compiled by the JMA (available online at http://www.jma.go.jp/jma/jma-eng/ jma-center/rsmc-hp-pub-eg/besttrack.html), for which the position, size, and strength of typhoons over the western North Pacific from genesis to lysis are archived at a typical sampling interval of $6 \mathrm{~h}$.

In section 6, we also apply the NEAT algorithm to the 6-hourly output of a 10-yr preindustrial control run of an atmosphere-ocean coupled GCM, the Model for Interdisciplinary Research on Climate, version 3.2 (high resolution) [MIROC3.2 (hires)], ${ }^{2}$ which has a higher resolution than JRA-25/JCDAS. The model was run with a horizontal resolution of T213 $(\sim 50 \mathrm{~km})$ and 56 vertical levels in the sigma coordinate. See Sakamoto et al. (2012) for more details of the model configuration and performance.

\section{Methodology}

\section{a. Neighbor enclosed area tracking}

NEAT is a new cyclone tracking method based on connecting several areas, which is different from conventional

\footnotetext{
${ }^{1}$ The domain is monitored for typhoons by the JMA.

${ }^{2}$ MIROC was jointly developed by the Atmosphere and Ocean Research Institute of The University of Tokyo, the National Institute for Environmental Studies of Japan, and the Research Institute for Global Change of the Japan Agency of Marine-Earth Science and Technology.
}

point-to-point tracking methods [see Williams and Houze (1987), Arnaud et al. (1992), Mathon and Laurent (2001), and Morel and Senesi (2002) for descriptions of similar methods used for cloud detection and tracking]. The NEAT scheme simply entails equivalent labeling for spatiotemporally connected domains (Fig. 1). A series of equivalently labeled areas can be regarded as a track in the NEAT algorithm, and the track line can be drawn by connecting the centroids of equivalently labeled areas (Hodges 1994). The NEAT algorithm identifies, for every time frame, an enclosed area that satisfies the requirement of an intensity greater than the threshold $C$ with a relative vorticity of $850 \mathrm{hPa}$ and a size greater than $A$. The algorithm then assigns an equivalent label to the two enclosed areas that overlap in an area larger than $O$ between adjacent time frames. Tracks with a lifetime less than $H$ hours are discarded. The conditions $C, H, A$, and $O$ are interacting; therefore, we tune only $C$ in section 4 by setting $H=48 \mathrm{~h}, A=20000 \mathrm{~km}^{2}$, and $O=$ $10000 \mathrm{~km}^{2}$ (Fig. 1, Table 1). See appendix A for a test of the sensitivity to $A$ and $O$ and of how well the overlap method relieves the grid system dependency.

In the NEAT algorithm, TC identification is performed as a post-tracking step. The TC here should satisfy the vertical-shear condition $V$ and the warm-core temperature condition $W$. Although these conditions do not have to be satisfied contiguously, they should be satisfied for more than $H$ hours in total over the lifetime of the TC. Conditions $V$ and $W$ have the same physical meaning as the presence of a warm core, but they were named differently in this paper. Condition $V$ is satisfied when the speed of the maximum $850-\mathrm{hPa}$ meridional wind is larger than the maximum $300-\mathrm{hPa}$ meridional wind in the labeled area detected by NEAT. Condition $W$ is satisfied when the maximum $500-\mathrm{hPa}$ temperature anomaly ${ }^{3}$ in the labeled area is found in a cyclone circle with a radius $d$. Both conditions are important for eliminating extratropical cyclones with baroclinic instability structures and are tuned in section 4.

In section $7 \mathrm{~b}$, we attempt to estimate the extratropical transition timing by using the oblateness measure provided by the NEAT algorithm (Inatsu 2009; Inatsu and Amada 2013). By transforming to two-dimensional polar coordinates with the origin at the center of the enclosed area, ${ }^{4}$ the distance between the center and the

\footnotetext{
${ }^{3}$ If the one-level temperature at $500 \mathrm{hPa}$ were replaced with the vertically integrated temperature, the result would not change significantly. We decided to use the one-level temperature for the optimal TC identification because of its simplicity.

${ }^{4}$ Although the projection is distorted in the polar region, the shape estimation is relatively unaffected by the projection for cyclones that stretch over hundreds of kilometers and are typically south of $40^{\circ} \mathrm{N}$
} 


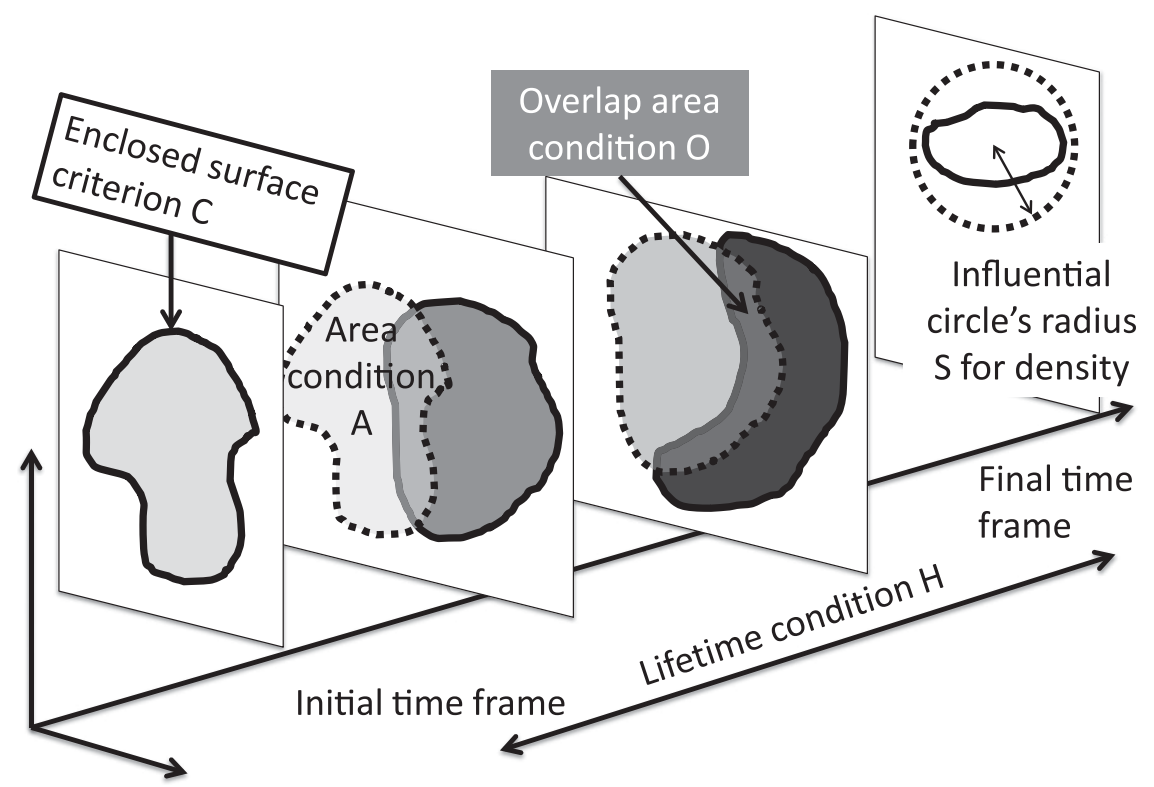

FIG. 1. Schematic of the neighbor enclosed area tracking (NEAT) algorithm and its tuning parameters in the spatiotemporal space; time increases to the top right, east is the direction of the bottom right, and north is upward. A cyclone defined as an enclosed surface image in the NEAT algorithm moves eastward from the initial to final timeframes in this schematic. The solid line denotes the boundary of the enclosed image in a timeframe, and the dotted line indicates the boundary in the previous timeframe. Condition $C$ is the criterion of 850 -hPa relative vorticity to identify enclosed surfaces, condition $A$ is the criterion of the enclosed surface size, condition $O$ is the criterion of the overlap size, and condition $H$ is the lifetime criterion. The influential circle's radius $S$ is used for calculating the cyclone density.

boundary of the labeled area is calculated as a function of the angle $\theta$ :

$$
r(\theta)=a_{0}+\sum_{n} a_{n} \cos n \theta+b_{n} \sin n \theta,
$$

where $a_{n}$ and $b_{n}$ are Fourier coefficients. Fitting the boundary shape to an ellipse given by $\tilde{r}=a_{0}+a_{2} \cos 2 \theta+$ $b_{2} \sin 2 \theta$ with a semimajor axis of $a=a_{0}+\sqrt{a_{2}^{2}+b_{2}^{2}}$ and a semiminor axis of $b=a_{0}-\sqrt{a_{2}^{2}+b_{2}^{2}}$, the oblateness of the enclosed area is then defined as $f=(a-b) / a$ in the NEAT algorithm.

\section{b. Neighbor point tracking}

An NPT algorithm is set up for comparison with the NEAT algorithm in sections 5 and 6 . The NPT first finds a point that satisfies the intensity condition $C^{\prime}$, which is that a local maximum of the 850 -hPa relative vorticity is greater than a tunable threshold, and identifies that point as a storm-center candidate. The estimated point is then connected to the nearest maximum point in the next time frame if the distance between them is less than $300 \mathrm{~km}$. The NPT estimates the storm center point in the next time frame as

$$
\mathbf{p}_{e}\left(t+t_{0}\right)=\mathbf{p}(t)+\left[\mathbf{p}(t)+\mathbf{p}\left(t-t_{0}\right)\right]
$$

where $\mathbf{p}$ is the position vector as a function of time and $t_{0}$ is $6 \mathrm{~h}$. Note that $\mathbf{p}_{e}$ is equal to $\mathbf{p}(t)$ at the genesis point. In the previous studies that used an NPT method, the temporal connection parameters were adjusted for fast background flow, but we simply assume a linear uniform motion on temporal connections.

The TC identification algorithm at the post-NPT tracking stage was performed with the vertical wind shear condition $V^{\prime}$ and the warm-core temperature condition $W$,

TABLE 1. Optimal parameter set for TC selection and tracking for the neighbor enclosed area tracking (NEAT) and neighbor point tracking (NPT) algorithms.

\begin{tabular}{lcc}
\hline \multicolumn{1}{c}{ Conditions for tracking } & NEAT & NPT \\
\hline$C$ (vorticity) & $110 \times 10^{-6} \mathrm{~s}^{-1}$ & $100 \times 10^{-6} \mathrm{~s}^{-1}$ \\
$H$ (lifetime) & 2 days & 2 days \\
$A$ (area) & $200 \times 10^{3} \mathrm{~km}^{2}$ & N/A \\
$O$ (overlap) & $100 \times 10^{3} \mathrm{~km}^{2}$ & N/A \\
$C$ onditions for TC selection & NEAT & NPT \\
$V$ (vertical shear) & On & On \\
$W$ (warm core) & $200 \mathrm{~km}$ & $240 \mathrm{~km}$ \\
\hline
\end{tabular}


and each had to be satisfied for longer than $H$ hours over the TC lifetime. To compare the NPT algorithm and the NEAT algorithm as fairly as possible, the TC identification component was kept as consistent as possible. The vertical-shear condition was satisfied when the maximum $850-\mathrm{hPa}$ meridional wind was stronger than the maximum $300-\mathrm{hPa}$ meridional wind in the cyclone circle, which had a radius of $300 \mathrm{~km}$. The difference between conditions $V$ and $V^{\prime}$ is the choice of area used to search the vertical shear, but this difference is not likely to introduce large discrepancies into the results because the size of TCs ranges between 100000 and $300000 \mathrm{~km}^{2}$ (see Fig. 9), which corresponds to a circle with a radius of between 200 and $300 \mathrm{~km}$. We apply the exact same warm-core condition as in the NEAT method. These parameters are optimized in section 4.

\section{c. Tracking comparison}

A set of track lines is compared in sections 5, 6, and 7b and appendix C. A common procedure is required for calculating the track density from track line information data given as a time sequence of longitudes and latitudes. For each track line $k$ for which the distance from the track line segment is less than the geodesic distance of $300 \mathrm{~km}$, the weight $d_{k}(x, y)$ is set to 1 ; elsewhere, $d_{k}(x, y)=0$. The track density is defined as $D=\sum_{k} d_{k}(x, y)$. Similarly, the genesis density is defined as $G=\sum_{k} g_{k}(x, y)$. Here, for each track $k$ for which the distance from a genesis point is less than the geodesic distance of $300 \mathrm{~km}$, the weight $g_{k}(x, y)$ is set to 1 ; elsewhere, $g_{k}(x, y)=0$. This definition is quite intuitive (and the units are $\mathrm{m}^{-2}$ ), but the definition only yields nonnormalized statistics, similar to the constant kernel method in Hodges (1996).

To match the results with the best track data in section 5 , we define two tracks as matched when the distance between the typhoon center in the best-track data and the track point obtained with the algorithm remains less than $300 \mathrm{~km}$ for more than 1 day. The situation is called a hit or a miss when an algorithm captures or loses a typhoon in the best-track data, respectively, and is called a false alarm when an algorithm-based track does not match any of the typhoons in the best-track data. Thus, misses and false alarms correspond to type I and type II errors, respectively, for the null hypothesis that an algorithm can capture a TC correctly. We evaluate the performance of the algorithm on the basis of the hit ratio and the total number of errors, which is defined as the sum of misses and false alarms.

\section{Tuning}

Here, we search for an optimal parameter set for NEAT and NPT in a bounded domain of the three-dimensional parameter space that is spanned by the intensity condition $C$ (or $C^{\prime}$ ), the vertical-shear condition $V\left(\right.$ or $\left.V^{\prime}\right)$, and the warm-core condition $W$. Regarding the intensity condition $C$ ( or $C^{\prime}$ ), the vorticity thresholds considered range is between $80 \times 10^{-6}$ and $140 \times 10^{-6} \mathrm{~s}^{-1}$. The vertical-shear condition $V$ (or $V^{\prime}$ ) is tested by switching it on and off. The condition $W$ searches for a warm core by determining the location of the maximum temperature inside the TC, and we tuned the distance between the warm core and the cyclone center to be between 80 and $400 \mathrm{~km}$. The vertical-shear condition $V$ has essentially the same physical meaning as the warm-core condition $W$, but for practical reasons, we use both to obtain reasonable performance (see appendix B for a parameter sweep performed without condition $W$ using the NEAT tracking). As the conditions become weaker, the ratios of hits and misses approach $100 \%$ and $0 \%$, respectively, but the number of false alarms increases. We are currently investigating a parameter set that yields the lowest number of errors in this parameter space.

Figure 2a shows the hit ratio and the total number of errors in the case when the vertical-shear condition is switched on for the tuning of the NEAT algorithm. The hit ratio is quite sensitive to the warm-core tuning parameter in domains of smaller than $200 \mathrm{~km}$; everywhere else, it is sensitive to the intensity condition instead. This result indicates that the warm-core condition loses its effectiveness if the warm core is sought in too large a cyclone circle. The minimum number of errors is $6.90 \mathrm{yr}^{-1}$ for an intensity parameter of $110 \times 10^{-6} \mathrm{~s}^{-1}$ and a warm-core parameter of $200 \mathrm{~km}$. The NEAT algorithm with this optimal parameter set can capture approximately $86 \%$ of the total best tracks with 3.83 misses and 3.07 false alarms per year (Fig. 2b). We present the results obtained with the NEAT algorithm with this parameter set after the next section. With the vertical-shear condition switched off, the total number of errors increases drastically over a broad area in the parameter space (Fig. 2c) because of the numerous false alarms (Fig. 2d). This result indicates that the verticalshear condition effectively excludes extratropical cyclones and reduces the frequency of false alarms.

Figure 3 shows the hit ratio and the total number of errors in the case when the vertical-shear condition is switched on for the tuning of the NPT algorithm. The hit ratio is quite sensitive to the warm-core tuning parameter in domains of size less than $300 \mathrm{~km}$; everywhere else, it is sensitive to the intensity condition instead. The minimum number of errors is $7.00 \mathrm{yr}^{-1}$ for an intensity parameter of $100 \times 10^{-6} \mathrm{~s}^{-1}$ and a warm-core parameter of $240 \mathrm{~km}$. The NPT algorithm with this optimal parameter set can capture approximately $88 \%$ of the total best tracks with 3.03 misses and 3.97 false alarms per 
(a) NEAT: hit rate and total error

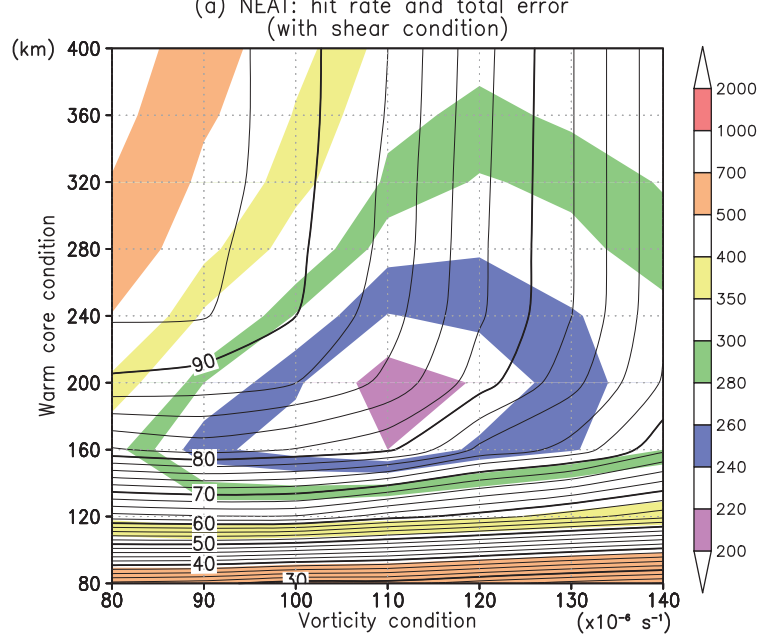

(c) NEAT: hit rate and total error

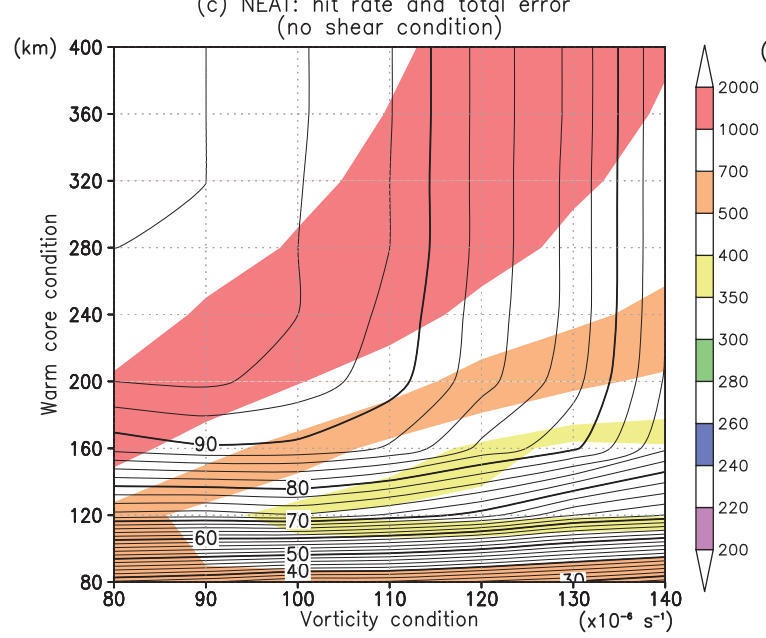

(b) NEAT: misses and false alarms

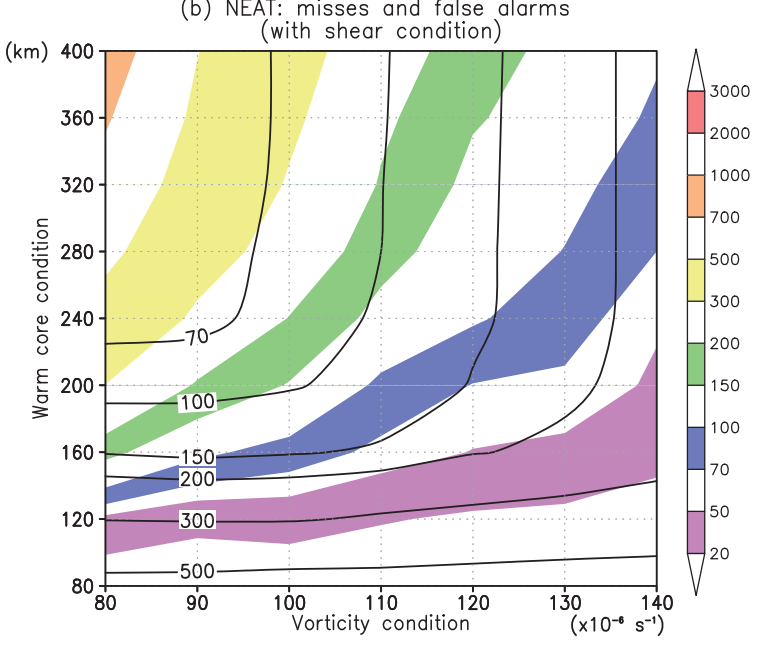

(d) NEAT: misses and false alarm

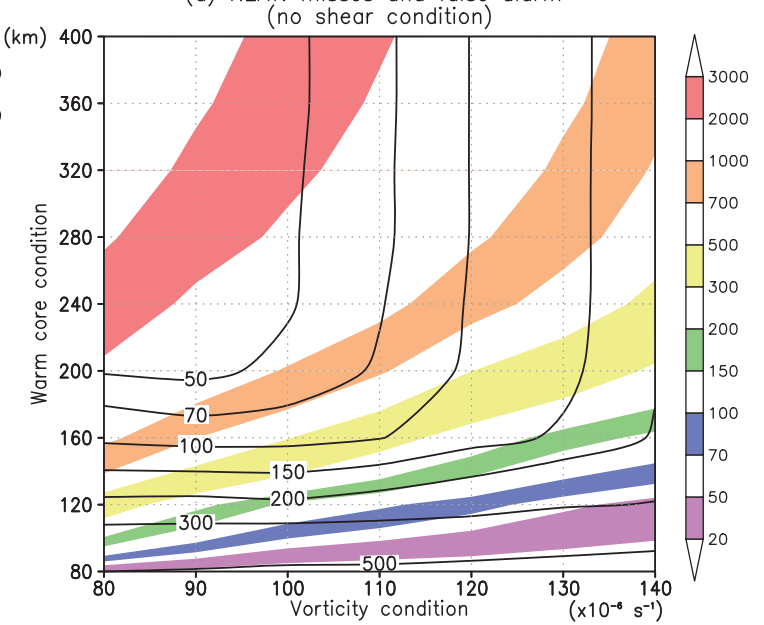

FIG. 2. (a) Hit rate (\%, contours) and total error amount (shaded areas), defined as the sum of misses and false alarms, in the twodimensional plane spanned by two parameters-the vorticity condition in the horizontal direction and the warm-core condition in the vertical direction-in the tropical cyclone (TC) tracking by the NEAT algorithm using the wind shear condition. See the text for the details of the conditions. The contour interval is $2 \%$, and the shading is per the legend on the right. (b) The number of misses (contours) and false alarms (shaded areas) in the parameter plane. (c),(d) As in (a),(b), but for the NEAT algorithm without the wind shear condition.

year (Fig. 3b). We present the results of the NPT algorithm with this parameter set after the next section. The vertical-shear condition also effectively excludes extratropical cyclones in the NPT algorithm (not shown). Hatsushika et al. (2006) demonstrated that in their NPT algorithm, the TC detection rate for JRA-25 is approximately $83 \%$ for data for the western North Pacific between 1990 and 1996. Their algorithm can obtain as many TCs as our NPT algorithm.

Summarizing the above results, we obtain the optimal parameter set of $(C, V, W)=\left(110 \times 10^{-6} \mathrm{~s}^{-1}\right.$, switched on, $200 \mathrm{~km})$ for NEAT and $\left(C^{\prime}, V^{\prime}, W\right)=\left(100 \times 10^{-6} \mathrm{~s}^{-1}\right.$, switched on, $240 \mathrm{~km}$ ) for NPT (Table 1). Conditions $C$ and $C^{\prime}$ are different because the intensity condition is attributed to the temporal connection method. Conditions
$V$ and $V^{\prime}$ are slightly different in terms of the definition of the domain, and the thresholds in condition $W$ are also slightly different, as described above. The results obtained with the NEAT method with the optimal parameter set $(C, V, W)$ are as accurate as those obtained with NPT with the optimal parameter set $\left(C^{\prime}, V^{\prime}, W\right)$. We now use the two different optimal parameter sets for a comparison between the two algorithms, but this choice does not significantly affect the results.

\section{Performance}

The climatological annual-mean track density for NEAT and NPT is $15 \%$ lower than the observed typhoon density (Fig. 4). The NEAT algorithm captures 
(a) NPT: hit rate and total error

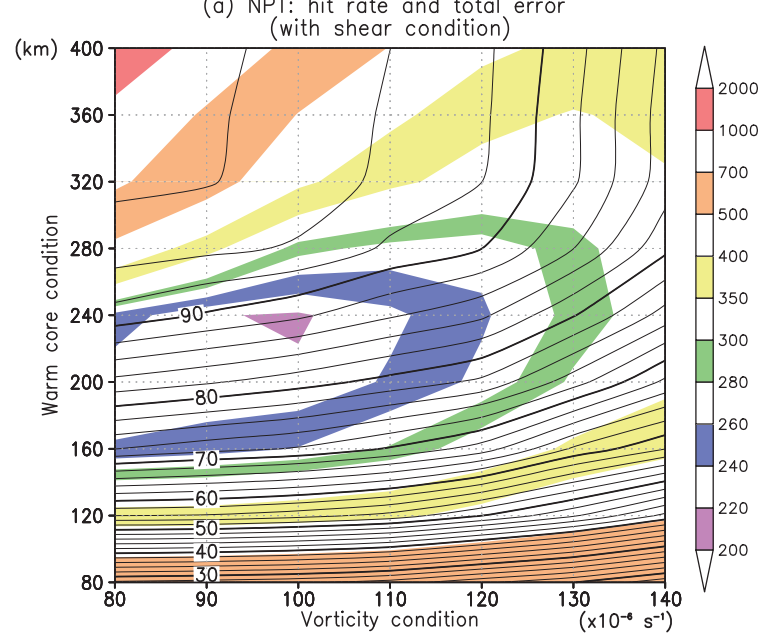

(b) NPT: misses and false alarms

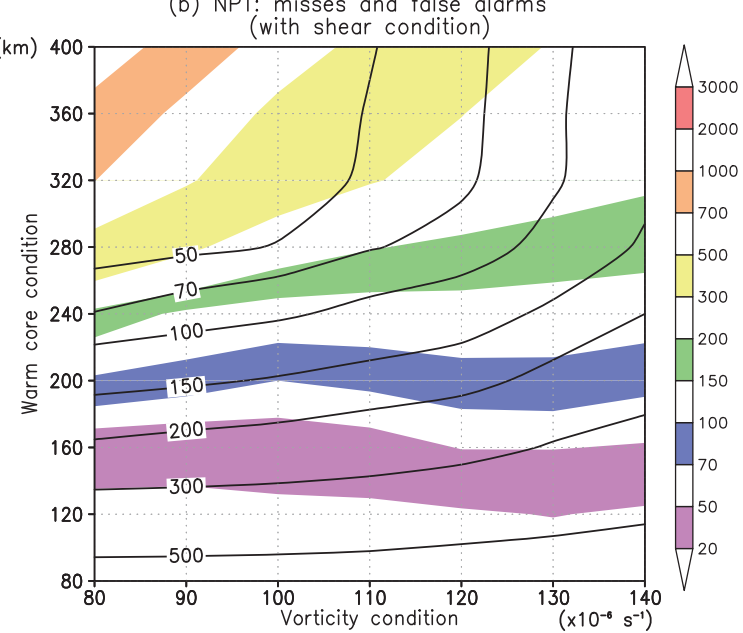

FIG. 3. As in Fig. 2(a),(b), but for TC tracking by the neighbor point tracking (NPT) algorithm.

the spatial distribution of the observed typhoon tracks, which were frequently generated to the east of the Philippines and moved toward the region south of Japan. North of $40^{\circ} \mathrm{N}$, the NEAT algorithm was unable to completely remove extratropical cyclones (see appendix $\mathrm{C}$ for more details). The NPT algorithm also accurately captured the spatial distribution in the western North Pacific but slightly underestimated the number of cyclones in the midlatitudes. More sophisticated NPTs could improve this underestimation. The NEAT and NPT algorithms also correctly estimate the genesis areas in the east and west of the Philippines (Fig. 5).

Because the observed cyclones are accurately captured by both methods, seasonal variability (Fig. 6) and interannual variability (Fig. 7) are also expected to be captured accurately, which is indeed the case (Figs. 6 and 7). As for interannual variability, during the warm and cold events in the El Niño-Southern Oscillation (ENSO), the annual-mean TC track density yielded by both algorithms closely reproduces the spatial distribution of the observed typhoon track density (Fig. 7). Here, we selected the warm events in 1982, 1987, 1991, 1997, and 2002 and the cold events in 1984, 1985, 1988, 1999, 2000, and 2007. Warm events expand the range for typhoon tracks to cover the entire tropical North Pacific region because of the weak Bonin high, whereas cold events limit tracks to the region between the Philippines and Japan (Wang and Chan 2002; Chan and Liu 2004). This tropical cyclone response to ENSO can be found in tracks by NEAT and NPT algorithms, but our NPT algorithm slightly misses the extratropical part (Figs. 7e,f). This could be improved by more sophisticated NPT algorithms.

\section{Grid system dependence}

This section discusses the dependence of TC tracking on the dataset's grid system. As discussed in the introduction, the NEAT algorithm is expected to reduce the dependence of the tracking process on the grid system, whereas NPT requires an additional process to unify the grid systems of the datasets considered. Here, we prepare two identical datasets with different grid systems for comparison. A dataset with a coarser grid is created by aggregating surrounding grids; the grid mesh of the resulting dataset is still sufficiently fine for capturing TCs. The grid system of the JRA-25/JCDAS data is sufficiently fine for capturing TCs; however, the grid of the aggregated dataset is too coarse. A successful example of tracking Typhoon Songda is presented in appendix A, but there were many missed TCs (not shown). In this section, we demonstrate that the NEAT algorithm can be applied to model outputs with the same parameter set tuned on the basis of analysis data with different grid spacing. Therefore, we chose the MIROC GCM output with a T213 horizontal grid mesh $(\sim 60 \mathrm{~km})$. The dataset with the aggregated coarser grid was created using a 1-2-1 low-pass filter for the zonal and meridional directions, resulting in data with an approximately T106 grid mesh $(\sim 110 \mathrm{~km})$, which is comparable to that of JRA-25/JCDAS. We maintain the optimal parameter sets (Table 1) for tracking with the original and aggregated datasets.

The spatial distribution of the climatological TC track density for the NEAT and NPT algorithms is shown in Fig. 8. The NEAT algorithm identified 368 TCs from the original-mesh data and 325 from the aggregated data. The TC tracks captured by the NEAT algorithm based 
(a) Annual-mean TC density (best track)

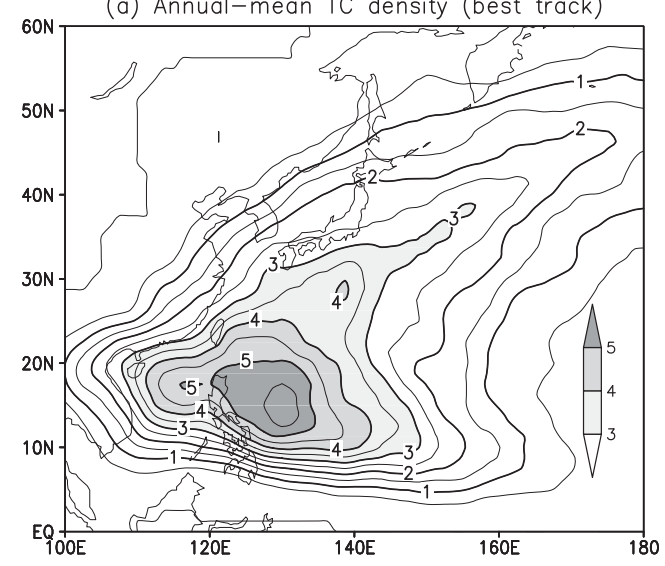

(b) Annual-mean TC density (NEAT track)

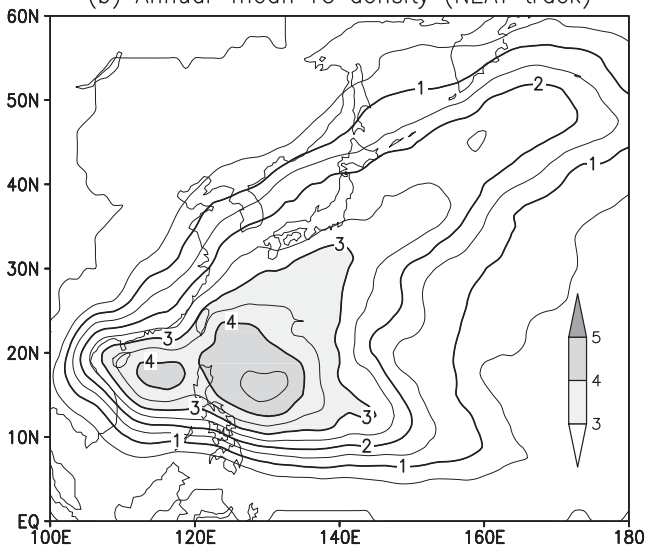

(c) Annual-mean TC density (NPT track)

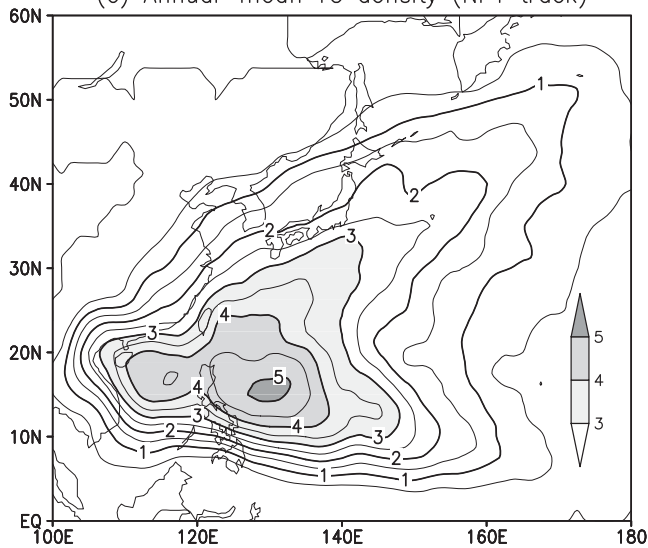

FIG. 4. Climatological annual-mean track density for (a) typhoons in the best-track data published by the Japan Meteorological Agency (JMA) and tracks estimated by using the (b) NEAT-based and (c) NPT-based TC tracking algorithms to reanalyze the JRA-25/ JCDAS data. The contour interval is $0.5 \mathrm{yr}^{-1}$, and the shading is per the legend on the bottom right in each panel.

on the original dataset extend from north of the Philippines to northeast of Japan (Fig. 8a). In the results based on the aggregated dataset, the tracks maintain the same spatial distribution, albeit with a slight and (a) Annual-mean TC genesis density (best track)

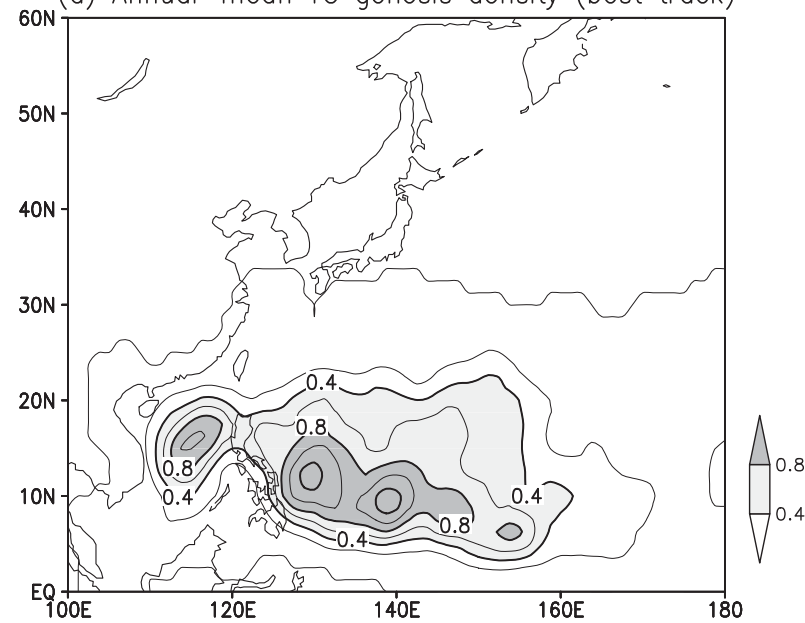

(b) Annual-mean TC genesis density (NEAT track)
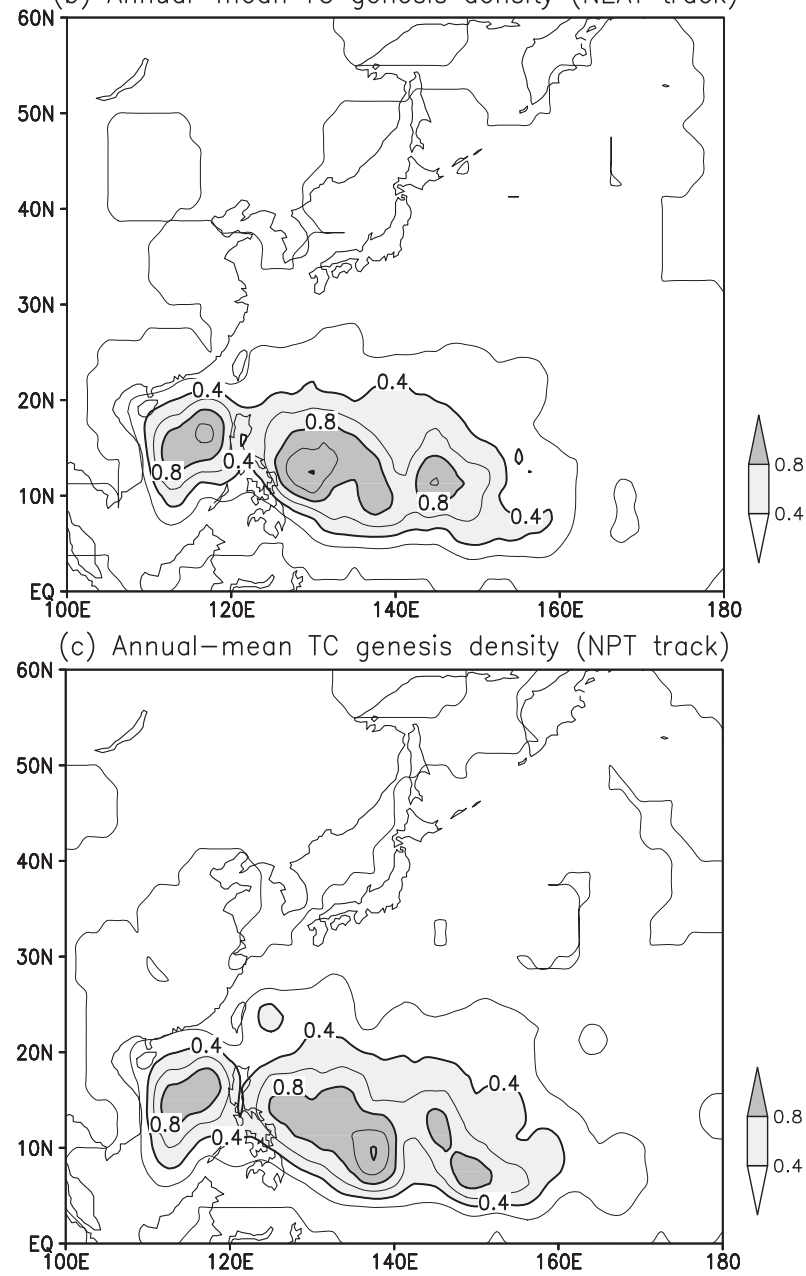

FIG. 5. As in Fig. 4, but for genesis density The contour interval is $0.2 \mathrm{yr}^{-1}$. 


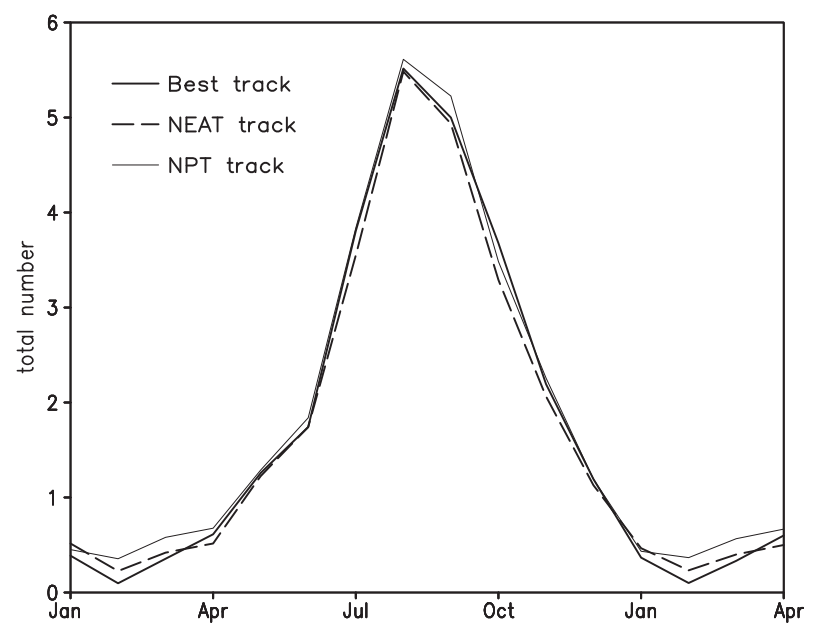

FIG. 6. Seasonal variation in the number of typhoons $\left(\right.$ month $^{-1}$ ) for the best-track data (solid line) and the number of TCs estimated with the NEAT-based (dotted line) and NPT-based (thin line) algorithms. The season spans from January to April of the following year.

uniform reduction in number (Fig. 8b). In contrast, the NPT algorithm captured 1783 TCs from the original dataset, whereas tracking with the aggregated dataset drastically reduced this number to 401. Although the
NPT algorithm captured somewhat weaker TC tracks from the aggregated dataset (Fig. 8d) compared with NEAT (Fig. 8b), both algorithms yielded a similar spatial pattern in TC density because of the highly tuned grid used in JRA-25/JCDAS, which has grid spacing comparable to T106. The above simple examination demonstrates that the NEAT algorithm is relatively independent of the grid system. The NPT algorithm, in contrast, mitigates this problem by tuning parameters or by regridding datasets to a common grid system.

\section{Other diagnostics}

\section{a. Tropical cyclone size}

Figure 9a displays the frequency distribution of the size of TCs detected throughout the period, which are estimated by using the enclosed area identification in the NEAT algorithm. Although the result depends on criterion A, here, we set the criterion to the smallest value (section 3). Cyclones after the extratropical transition are excluded in this subsection (see section $7 \mathrm{~b}$ for methods for identifying extratropical transitions). The TC surface area ranges from 40000 to $300000 \mathrm{~km}^{2}$ for the majority of TCs, and the mean TC

(a) TC density in warm phase (Best track)

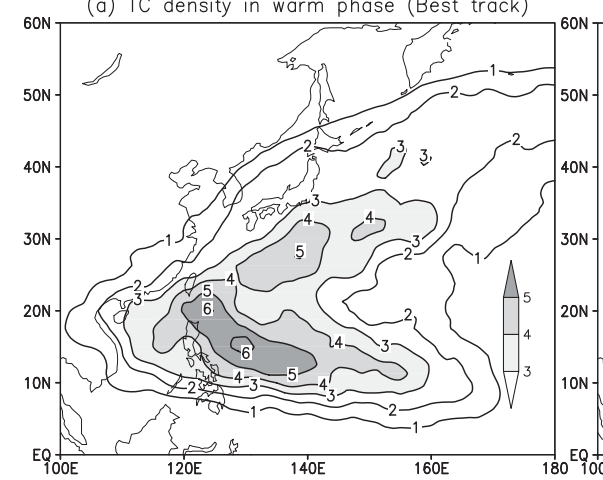

(d) TC density in cold phase (Best track)

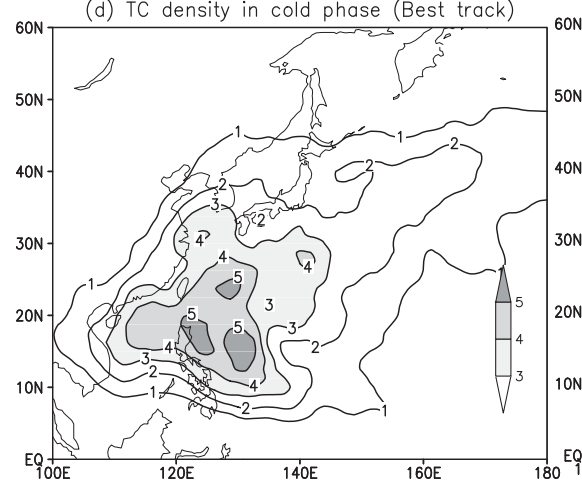

(b) TC density in warm phase (NEAT track)

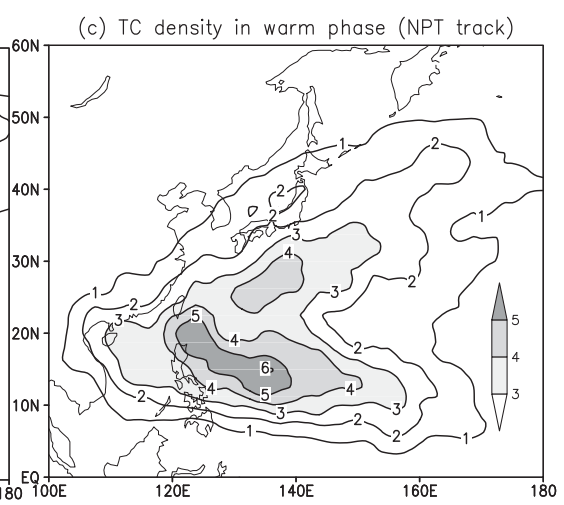

(e) TC density in cold phase (NEAT track)

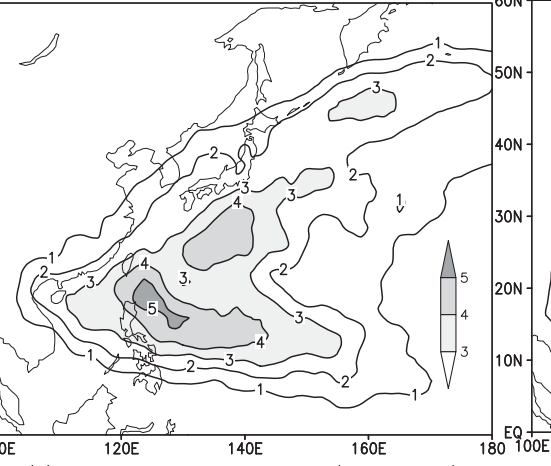

(f) TC density in cold phase (NPT track)
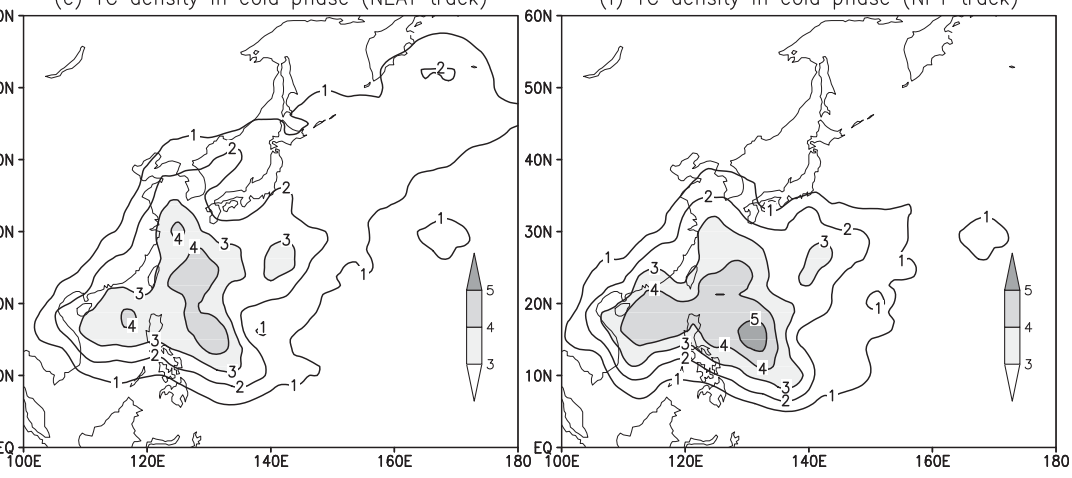

FIG. 7. (a) Annual-mean track density for observed typhoons in the warm years of El Niño-Southern Oscillation. The contour interval is $1 \mathrm{yr}^{-1}$, and the area in which the interval is $>3 \mathrm{yr}^{-1}$ is shaded. (b) Tracks for the NEAT algorithm and (c) for the NPT algorithm. (d)-(f) As in (a)-(c), but for the cold years. 
(a) NEAT: MIROC simulation with T213 grid

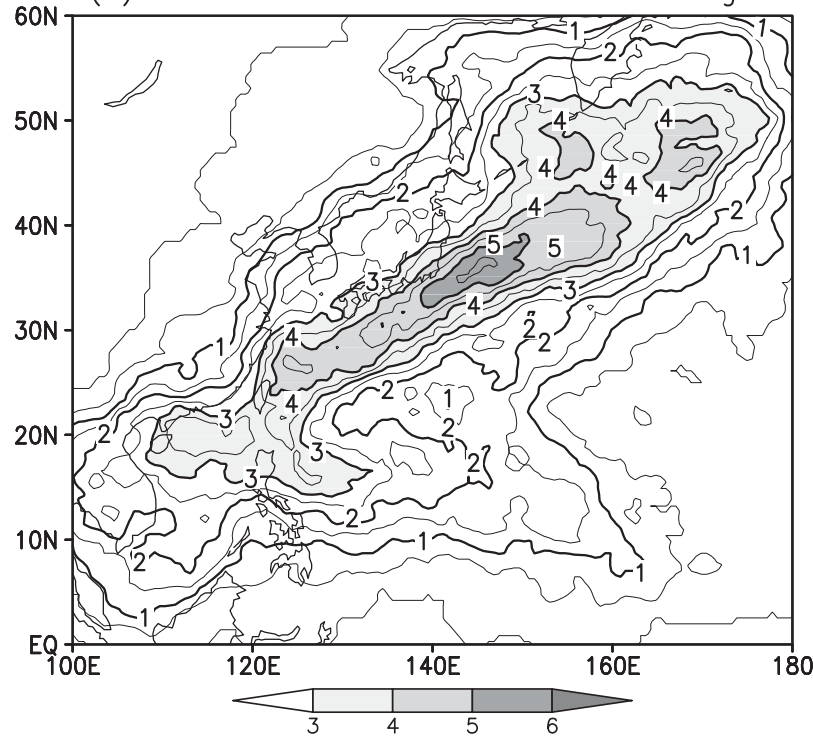

(c) NPT: MIROC simulation with T213 grid

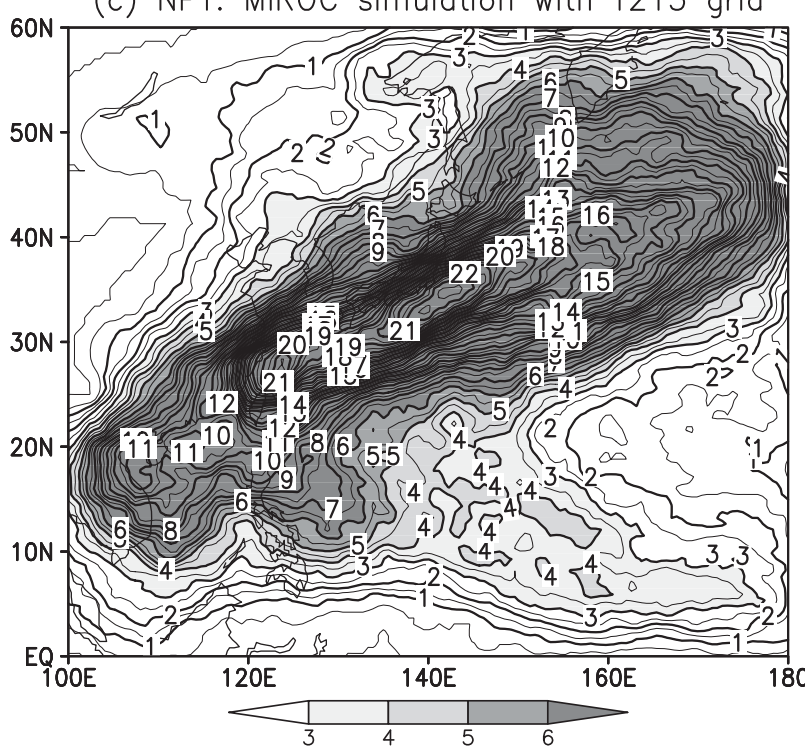

(b) NEAT: MIROC simulation with T106 grid

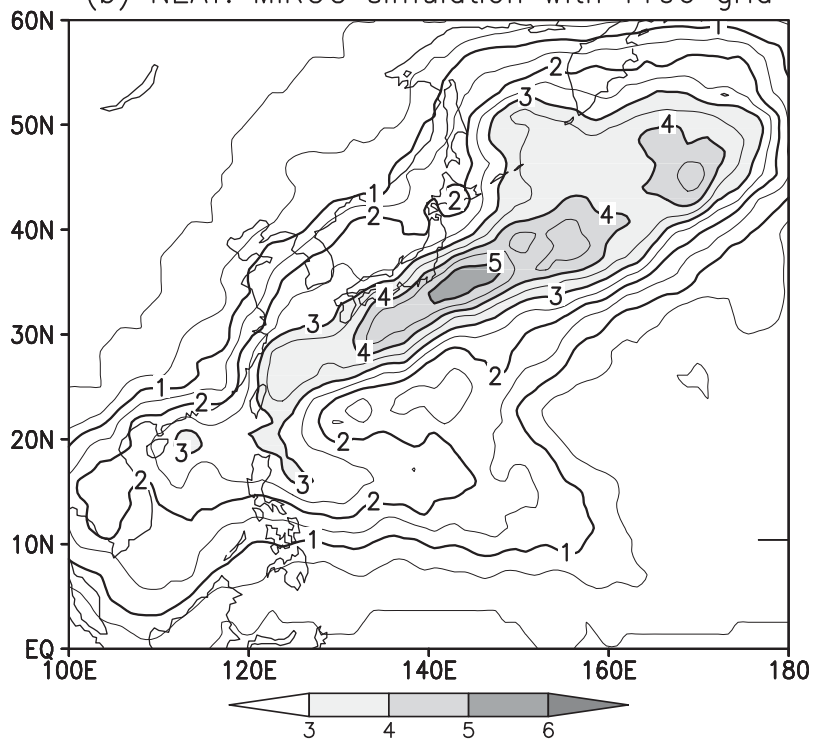

(d) NPT: MIROC simulation with T106 grid

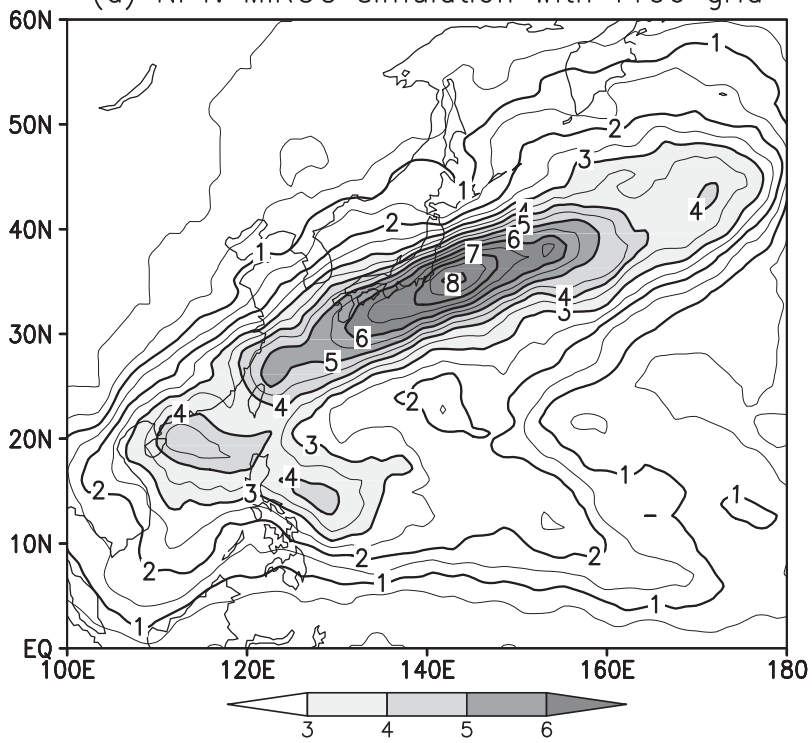

FIG. 8. Climatological annual-mean track density for tracks estimated by applying the NEAT-based TC tracking algorithm to (a) the original data and (b) the aggregate data; and by applying the NPT algorithm to (c) the original data and (d) the aggregate data. The original data are from a 10-yr prehistorical control simulation with a T213 horizontal resolution for the MIROC GCM. The contour interval is $0.5 \mathrm{yr}^{-1}$, and the shading is per the legend on the bottom.

size is approximately $200000 \mathrm{~km}^{2}$ (Fig. 9a). The frequency distribution is long tailed, and approximately $1 \%$ of TCs occupy areas greater than $500000 \mathrm{~km}^{2}$. Merrill (1984), who used the average radius of the outer closed isobar to estimate the size of TCs, demonstrated that the average area is $\sim 200000 \mathrm{~km}^{2}$ in the western North Pacific. Furthermore, the mean TC area is $\sim 260000 \mathrm{~km}^{2}$ according to Liu and Chan (1999) and $\sim 150000 \mathrm{~km}^{2}$ according to Chan and Yip (2003); these two estimates are based on a common definition of the average area with $15 \mathrm{~m} \mathrm{~s}^{-1}$ surface wind in the western North Pacific. Despite the fact that the TC size in the case of the NEAT algorithm is based on only a vorticity-threshold contour, the results reported here are consistent with those reported in previous studies.

The seasonal variability in size averaged over all TCs detected by the NEAT algorithm is shown in Fig. 9b, in which the number of TCs (Fig. 6) is superimposed. The mean TC size peaks in October, whereas the number of 

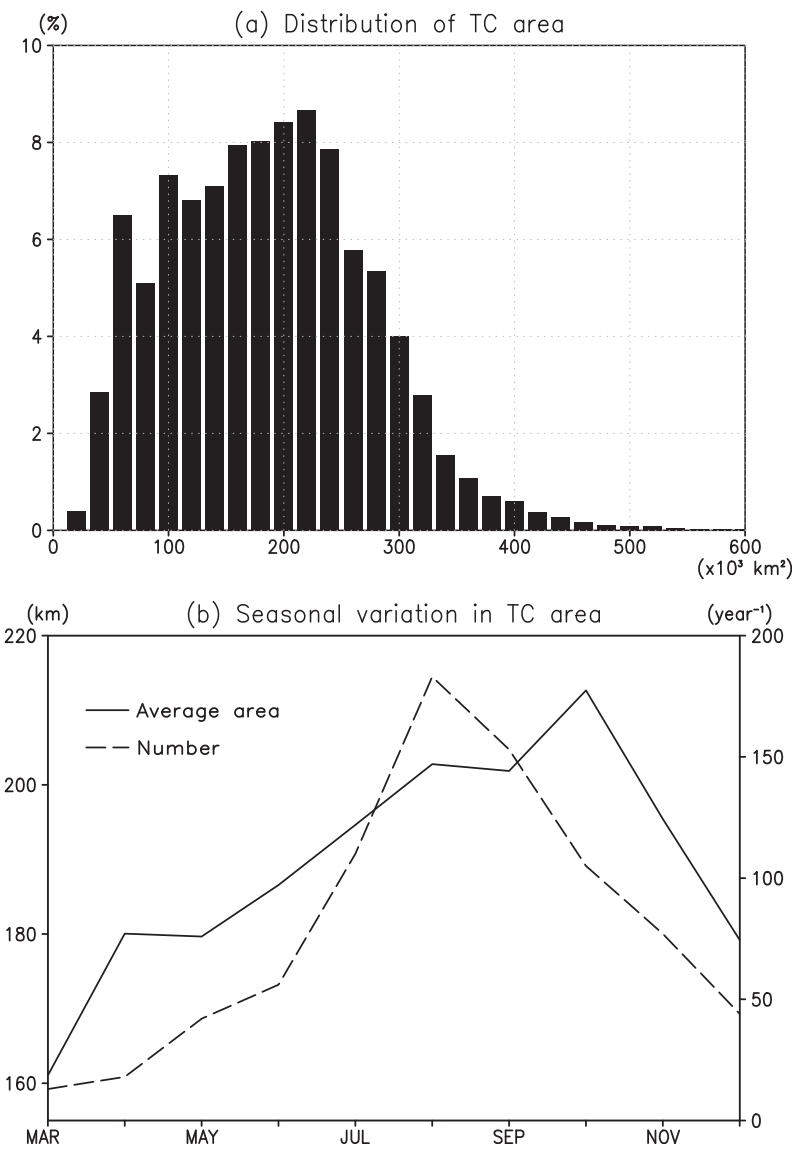

FIG. 9. (a) The frequency distribution (\%) of the TC area that the NEAT algorithm detected in the JRA-25/JCDAS data. The bin is set to $20000 \mathrm{~km}^{2}$. (b) Seasonal variation of the number of TCs estimated (solid line; scale on the right axis) and the average TC area (dotted line; scale on the left axis).

TCs peaks in August. The result is consistent with the findings of Brand (1972), Merrill (1984), Liu and Chan (1999), and Lee et al. (2010), who found a maximum mean TC size in October in the western North Pacific.

\section{b. Extratropical transition timing}

TCs often undergo extratropical transitions and change into baroclinic systems (Jones et al. 2003). TCs exhibit an axisymmetric structure with complex convective systems, ${ }^{5}$ whereas extratropical cyclones exhibit an asymmetric structure with baroclinic instabilities. Therefore, an extratropical transition should correspond to an axisymmetric distortion of an eddy and to

\footnotetext{
${ }^{5}$ Processes that are often highly nonlinear, including the cooperative intensification theory of Ooyama $(1969,1982)$, windinduced surface heat exchange (WISHE), and vertical hot-tower development, are also important.
}

TABLE 2. Number of hits, misses, and false alarms for extratropical transition tracks for the NEAT algorithm using the dynamical and geometric approaches.

\begin{tabular}{lcc}
\hline & Dynamical approach & Geometric approach \\
\hline Hits & 229 & 223 \\
Misses & 121 & 133 \\
False alarms & 159 & 103 \\
\hline
\end{tabular}

a reversal of vertical wind shear along the eddy periphery. Here, two possible approaches for detecting the time of an extratropical transition using the NEAT algorithm are employed. The first approach focuses on geometrical changes in the extratropical transition. Because the NEAT algorithm can provide an approximate estimate of the oblateness of the enclosed areas (section $3 a)$, an extratropical transition is identified when the 30-h running-mean oblateness during the TC life cycle exceeds 0.28 , which is equivalent to an ellipse with a ratio of semimajor axis to semiminor axis of approximately 4:3. The second approach focuses on dynamical changes in the extratropical transition. Because our TC detection algorithm includes the vertical wind shear condition in the enclosed area (section 3a), an extratropical transition is identified when this condition is violated (see Hart 2003).

The JMA subjectively recorded 364 typhoons between 1979 and 2009 that underwent an extratropical transition. Table 2 presents the number of hits, misses, and false alarms determined using the two approaches. The geometrical approach identified 223 extratropical transitions with 103 errors. This approach identified $59 \%$ of the identified TCs within $24 \mathrm{~h}$ of the average time difference. The mean time lag was approximately $24 \mathrm{~h}$. In comparison, the dynamical approach identified 229 extratropical transitions with 159 errors, and $61 \%$ of the identified TCs were within $24 \mathrm{~h}$ of the average time difference. The mean time lag was approximately $36 \mathrm{~h}$. It should be noted that the cyclone phase-space analysis performed by Kitabatake (2011) in the western North Pacific based on the same reanalysis dataset yielded slightly more accurate results, capturing approximately $75 \%$ of the extratropical transitions of TCs. The parts of tracks after an extratropical transition estimated by the geometrical approach are excluded from the analysis in sections $7 \mathrm{a}$ and $7 \mathrm{c}$.

\section{c. Meridional heat transport}

The NEAT algorithm can also be used to estimate the meridional heat transport of TCs. Meridional heat transport has been hypothesized to play a significant role in climate formation (Hart 2011), although this effect has not yet been directly demonstrated. Meridional heat 


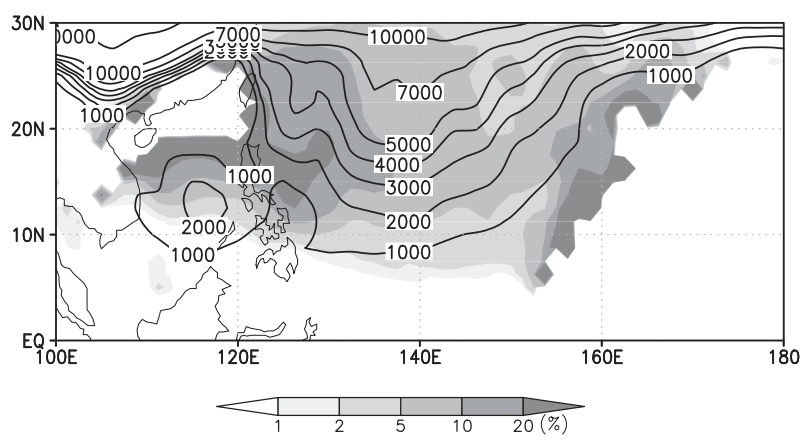

FIG. 10. The climatological meridional heat transport by the total transient eddies from June to November. The contours are at 1000 , $2000,3000,4000,5000,7000,10000$, and $20000 \mathrm{~kW} \mathrm{~m}^{-1}$. The ratio of the TC climatological meridional heat transport from June to November to the total (\%) is shown in grayscale at the bottom of the figure.

transport by transient eddies is evaluated following convention as

$$
M=\int_{z_{L}}^{z_{T}} \rho c_{p} \overline{v \theta} d z
$$

where $v$ is the transient-eddy meridional wind speed; $\theta$ is the transient-eddy potential temperature; $\rho$ is the air density; $c_{p}$ is the specific heat at constant pressure; $z_{L}$ and $z_{T}$ are set to the heights at 950 and $250 \mathrm{hPa}$, respectively; and the overbar indicates time-averaged values. A transient eddy is defined as the high-frequency part with a period of $<30$ days. We estimate the meridional heat transport due to TCs as

$$
M_{\mathrm{TC}}=\int_{z_{L}}^{z_{T}} \rho c_{p} \overline{v \theta H_{\mathrm{TC}}} d z
$$

where $H_{\mathrm{TC}}$, which is a support function of longitude, latitude, and time, equals 1 inside the NEAT enclosed area identified as a TC and 0 elsewhere.

The climatological meridional heat transport due to total transient eddies [Eq. (3)] and that due to TCs calculated by the NEAT algorithm [Eq. (4)], averaged from June to November, are shown in Fig. 10. TCs in the western North Pacific typically move along the western edge of the Bonin high and simultaneously transport heat inside the TC northward in a Lagrangian manner. Meridional heat transport is greatest near the Philippines. The TC meridional heat transport there is approximately $8.0 \times 10^{5} \mathrm{~kW} \mathrm{~m}^{-1}$. This transport corresponds to a single eddy that occupies an area for 7 days between June and November or an eddy with an anomalous internal temperature of $5 \mathrm{~K}$ that moves northward at a speed of $20 \mathrm{~km} \mathrm{~h}^{-1}$. Meridional heat transport by TCs near the Philippines also accounts for $10 \%-20 \%$ of the total for all transient eddies, which suggests that TCs in fact play a role in meridional heat transport in the subtropics in summer and autumn. In spring and winter, meridional heat transport by TCs is small because fewer TCs are generated, even in the western North Pacific.

\section{Conclusions}

We have proposed an alternative TC tracking method based on the NEAT algorithm, in which tracking is performed by overlapping an enclosed area between adjacent timeframes (Inatsu 2009; Inatsu and Amada 2013). The parameters of the intensity, vertical-shear, and warm-core criteria were intensively tuned by performing comparisons with observed typhoon best tracks, and the parameters were optimized to capture more than $85 \%$ of the typhoons from the JRA-25/JCDAS reanalysis dataset. This performance is as high as that of a conventional NPT algorithm, which was also intensively tuned in this study. The NEAT algorithm with this optimal parameter set tuned using the reanalysis data was applied to modern high-resolution GCM data, for which it successfully captured realistic TC statistics. Moreover, we demonstrated in this GCM examination that the NEAT algorithm was relatively independent of the dataset grid system.

The NEAT algorithm additionally provided realistic statistics for obtaining the size of TCs and for estimating extratropical transition timing and meridional heat transport due to TCs. The size of TCs was used in the original NEAT system; the average TC size was $200000 \mathrm{~km}^{2}$ in the western North Pacific, which is consistent with previous studies. Although the extratropical transition of TCs has previously been estimated objectively, we approached the problem by using either geometrical or dynamical characteristics of enclosed-area images yielded by the NEAT algorithm. We demonstrated that both methods provided results consistent with the subjective evaluation performed by the JMA. Meridional heat transport due to TCs was found to play a role in meridional heat transport in the western Pacific in summer and autumn.

The new algorithm may have applications in disaster evaluation. The TC axisymmetric circle is not precisely equal to the area damaged by the TC; the northeast part of the TC circle generally causes the greatest damage. The enclosed-area identification process in the NEAT algorithm can potentially identify the damage area using a measure of gale-force winds or heavy rainfall instead of relative vorticity. If we were to apply the NEAT algorithm to a warm-climate simulation employing a GCM, 
it would indicate areas in which floods, shipwrecks, landslides, and building destruction are likely. Our new algorithm could furthermore contribute to creating near-future hazard maps in countries in which typhoons and hurricanes are frequent, such as Japan and the United States. The accumulated cyclone energy (Bell et al. 2000) and the power dissipation index (Emanuel 2005) would be suitable measures for this purpose.

Although we propose an alternative TC tracking algorithm in this paper, the algorithm may have drawbacks, some of which are common to TC tracking in general. First, the NEAT method can miss fast-moving systems. When TCs undergo an extratropical transition, they can be entrained into the westerly flow and accelerate considerably. Extremely fast-moving extratropical cyclones occasionally move at speeds faster than $30 \mathrm{~m} \mathrm{~s}^{-1}$. A simple (fixed) overlap parameter is unlikely to solve this problem, and a variable threshold that depends on the background flow might solve this problem [see Denman et al. (2007) for an analogous example in an image-processing problem], although it drastically complicates the algorithm. Furthermore, although not discussed in this paper, computing the TC velocity depends on the choice of a system center. If the center is chosen to coincide with centroid, then the system velocity may not be estimated accurately for distorted systems. Because the NEAT algorithm can recognize the shape of images simultaneously with the tracking, the system velocity could be replaced with certain mapping quantities. For example, a fit to a quadratic form turns a temporal change into an affine transformation. However, this quadratic approximation would not always be valid because the cyclone shape is often too distorted and quite different from an ellipse. Such an evaluation of geometrical transformations is beyond the scope of this paper and remains an open question. Another problem is that the NEAT algorithm with fixed criteria $\mathrm{C}$ and $\mathrm{A}$, as in the optimal set (Table 1), was unable to capture smaller-scale TCs, such as those in the early stages. In contrast, in the study of Bengtsson et al. (2007), tracks were restricted to cases in which the TC criteria were fulfilled to obtain the closest agreement with the observed distributions, thereby determining much earlier and later stages of the TC life cycle. Regarding this point, there is the possibility that the NPT method is superior to NEAT; we will examine this possibility in a future study.

Acknowledgments. The authors thank the editor, the three anonymous reviewers, Prof. S. Minobe, Prof. M. Kimoto, Mr. K. Bessho, Dr. H. Hatsushika, Prof. H. Mukougawa, and Dr. Y. Miyamoto for their insightful comments. This study was partially supported by the
Program for Risk Information on Climate Change, the Research Program on Climate Change Adaptation, the Grant-in-Aid for Scientific Research on Innovative Areas 22106008, and the Grant-in-Aid for Scientific Research (A) 22244057, which are all funded by the Ministry of Education, Culture, Sports, Science, and Technology of Japan. The figures were created using Grid Application Development Software.

\section{APPENDIX A}

\section{Sensitivity of Area and Overlap Criteria}

This paper tuned only the vorticity criterion $C$ with TC-selection conditions $V$ and $W$, whereas the other criteria, $A, O$, and $H$, were fixed in the NEAT algorithm. Appendix A attempts to examine the sensitivity of the area and overlap criteria to guarantee the relevance of tuning by criterion $C$ as presented in this paper. Fixing criteria $C$ and $O$ to the optimal values (Table 1), the area criterion changes from $20 \times 10^{3}$ to $400 \times 10^{3} \mathrm{~km}^{2}$ (Fig. A1). It should be noted that the area criterion A should be larger than 2 grid cells $\left(\sim 20 \times 10^{3} \mathrm{~km}^{2}\right.$ in the subtropics) to track a slow-moving system. The hit rates and the total number of errors are similar to those obtained with the optimal parameter set with $A$ at $<60 \times$ $10^{3} \mathrm{~km}^{2}$; the hit rate decreases and the number of errors increase at larger $A$ values. The hit rate is less than $10 \%$ for $A>300 \times 10^{3} \mathrm{~km}^{2}$. This result is consistent with the size estimation (Fig. 9a), suggesting that most TCs in the western North Pacific range from $100 \times 10^{3}$ to $300 \times$ $10^{3} \mathrm{~km}^{2}$ in area. Moreover, this tuning property of $A$ indicates its similarity to vorticity condition $C$. However, as shown in Fig. 2, the sensitivity of condition $C$ appears to be somewhat lower, at least in the tuning range examined. Furthermore, condition $C$ can be freely set to any value, whereas the results are drastically changed with the area criterion $A$ near an integer multiple of a single gridcell size (not shown); thus, the hit rate is a continuous function of $C$ but a discrete function of $A$.

In contrast, we examine the sensitivity of $O$ with the optimal criterion $C$, as presented in Table 1. First, the overlap condition $O$ must be less than $A$; otherwise, condition $A$ is replaced with the value of condition $O$. The optimal value of $O$ of $10 \times 10^{3} \mathrm{~km}^{2}$ is the smallest, and there is no way to tune this parameter. Tuning $O$ at a larger $A$ of $100 \times 10^{3} \mathrm{~km}^{2}$ is attempted. However, criterion $O$ exhibits low sensitivity in this case, with hit rates and total number of errors as large as in the case of the smallest $O$ value (Fig. A2).

We expand the discussion on the overlap to demonstrate the relative independence with respect to the grid 

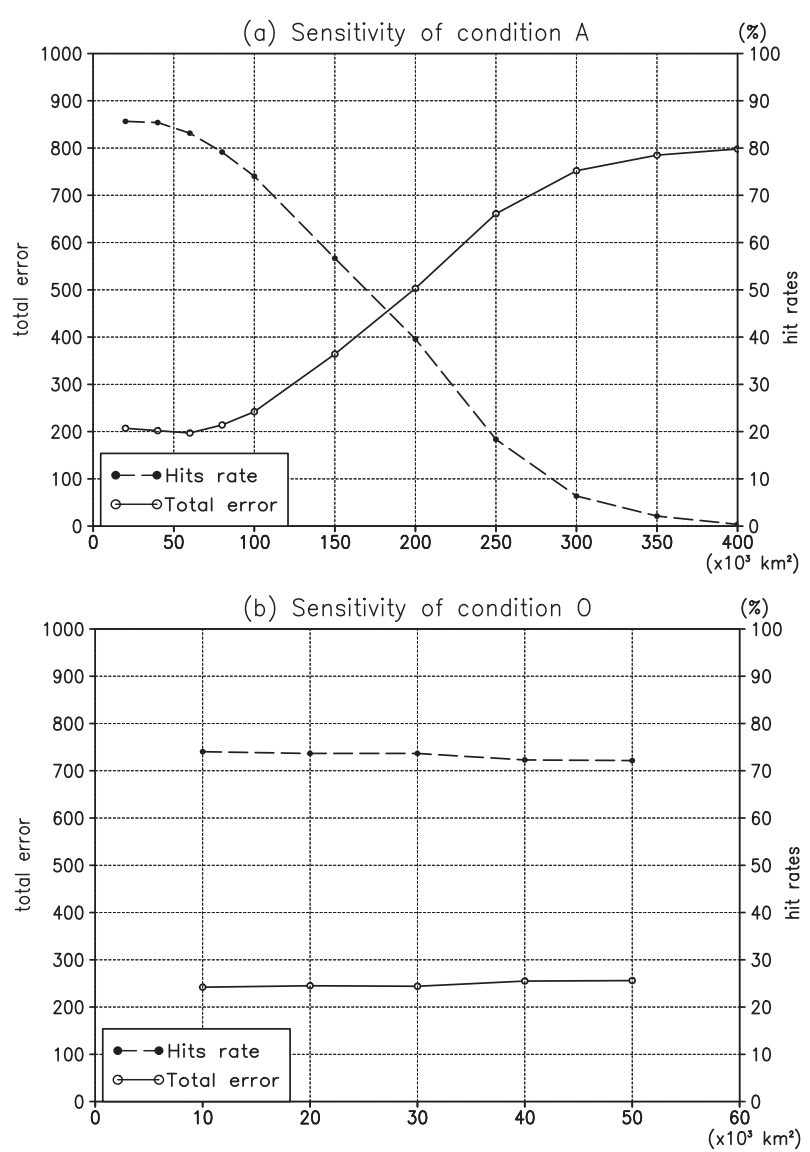

FIG. A1. (a) Hit rate (\%, right axis label, dashed line) and total error amount (left axis label, solid line) defined as the sum of misses and false alarms for tuning condition $A$ at a threshold $C$ of $110 \times$ $10^{-6} \mathrm{~s}^{-1}$ and a threshold $O$ of $10 \times 10^{3} \mathrm{~km}^{2}$, which are both fixed in the NEAT algorithm. (b) Hit rate and total error amount for tuning condition $O$ at a threshold $C$ of $110 \times 10^{-6} \mathrm{~s}^{-1}$ and a threshold $A$ of $100 \times 10^{3} \mathrm{~km}^{2}$, which are both fixed.

system of NEAT by examining a single-track example of Typhoon Songda, a long-lived typhoon that occurred between 19 August and 4 September 2004 (Fig. A2). The NEAT algorithm successfully reproduced the track of Songda based on the original JRA$25 / \mathrm{JCDAS}$ reanalysis data with $1.25^{\circ} \times 1.25^{\circ}$ grid spacing (Fig. A2a). In the subtropics, where the typhoon slowly moved westward, the enclosed-area image identified by NEAT algorithm (section 3a) in a timeframe almost fully overlapped the enclosed-area image in the next timeframe. The typhoon occupied 17 grid points both in 0000 and 0600 UTC 25 August, with 14 common grid points between these timeframes. However, in the extratropics, where the typhoon rapidly moved eastward after an extratropical transition, the enclosed-area images tended to partially overlap (Fig. A2c). The typhoon occupied 29 grid points in both 0000 and 0600 UTC 31 August, but there are only five

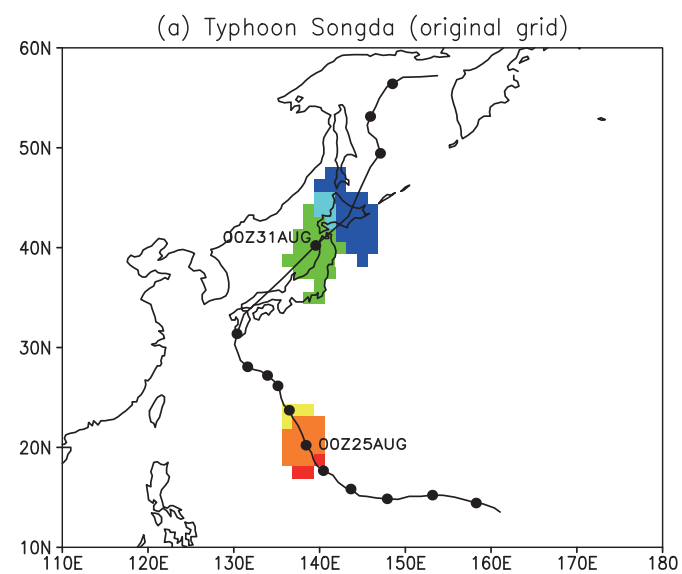

(b) Typhoon Songda (coarse grid)
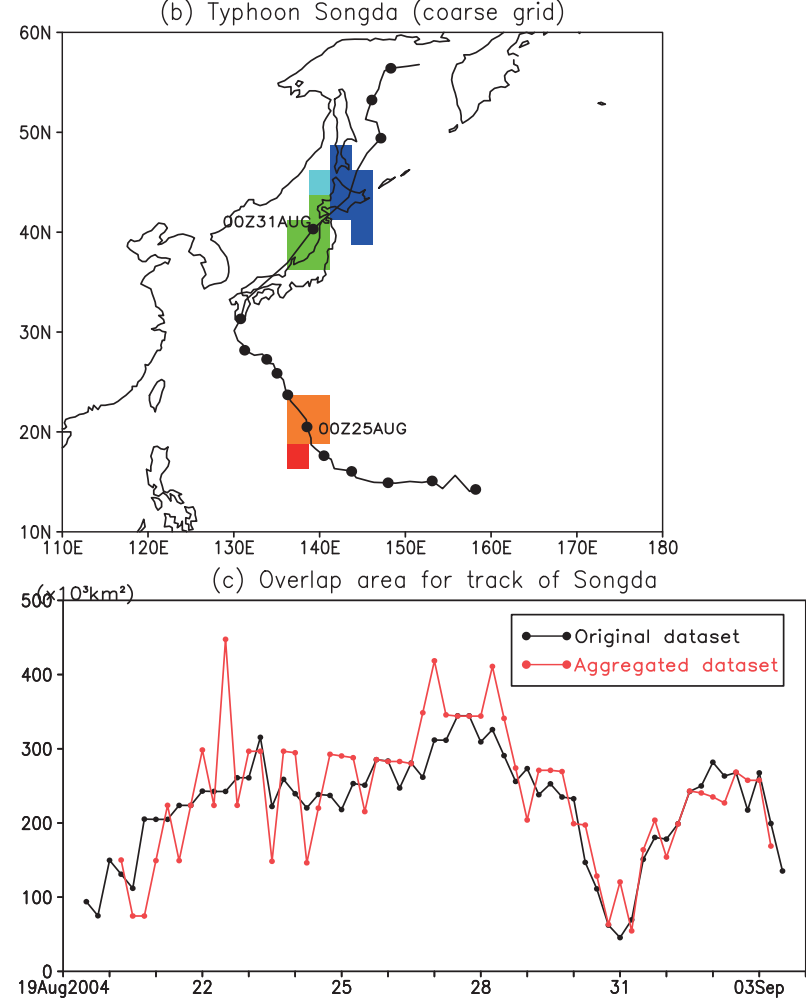

FIG. A2. Tracking of Typhoon Songda in 2004 by the NEAT algorithm based on (a) the original JRA-25/JCDAS reanalysis dataset and (b) the data aggregated using a spatial 1-2-1 low-pass filter. The line is the track of the centroid of the track image, and solid circles denote the position at 0000 UTC for each day. Red and yellow denote the enclosed-area image at 0000 and 0600 UTC $25 \mathrm{Aug}$, and orange indicates the overlap between them. Green and blue denote the enclosed-area image at 0000 and 0600 UTC 31 Aug, and sky blue indicates the overlap between them. (c) Time series of the overlap area $\left(10^{3} \mathrm{~km}^{2}\right)$ for the track of Songda, where black and red lines indicate the NEAT tracks based on the original and aggregated datasets, respectively.

common grid points between these timeframes. The speed exceeded $20 \mathrm{~m} \mathrm{~s}^{-1}$ at this moment; thus, the system moved much faster than the usual system in the extratropics. Testing the Songda track based on the 


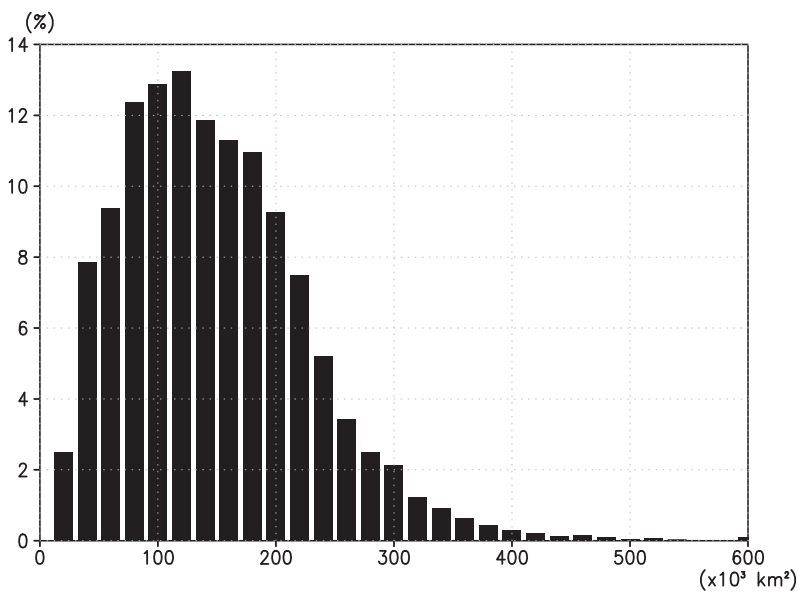

FIG. A3. The frequency distribution (\%) of the TC overlap area that the NEAT algorithm detected in the JRA-25/JCDAS data. The bin is set to $20000 \mathrm{~km}^{2}$.

coarse grid with $2.5^{\circ} \times 2.5^{\circ}$ grid spacing created using the spatial low-pass filter ${ }^{\mathrm{A} 1}$ applied to the original JRA-25/JCDAS data, the NEAT algorithm successfully reproduced the observed track, except for the generation stage. In the subtropical case on 25 August, there were four (coarse) grid points occupied at 0600 UTC, and these grid points were fully in common with the enclosed-area image in the previous timeframe at 0000 UTC. In the extratropical case on 31 August, there was only a single grid point that overlapped between the two adjacent timeframes. The overlap was hence maintained in the coarse grid data with slightly reduced accuracy. Examining the time series of overlap area during the life cycle of Songda, it fluctuated greatly in the coarse case and not in the original because the overlap value was quantized by a single grid cell.

Figure A3 displays the frequency distribution of the overlap area from May to October. Most of the overlaps ranged between 50000 and $250000 \mathrm{~km}^{2}$, but approximately $10 \%$ of the overlap areas were less than $50000 \mathrm{~km}^{2}$. Therefore, if the grid spacing were doubled to $2.5^{\circ} \times 2.5^{\circ}$, as in the above example, a few percent of the overlaps would not be realized because the minimum of overlaps is a single gridcell area of $\sim 60000 \mathrm{~km}^{2}$.

\section{APPENDIX B}

\section{Tuning by the Vertical-Shear Condition Only}

Appendix B demonstrates the necessity for the warmcore condition to obtain a reasonable performance of TC identification. We examined TC identification without

\footnotetext{
${ }^{\text {A1 }}$ The 1-2-1 low-pass filter is used for the zonal direction. Then, this filter is sequentially applied for the meridional direction.
}

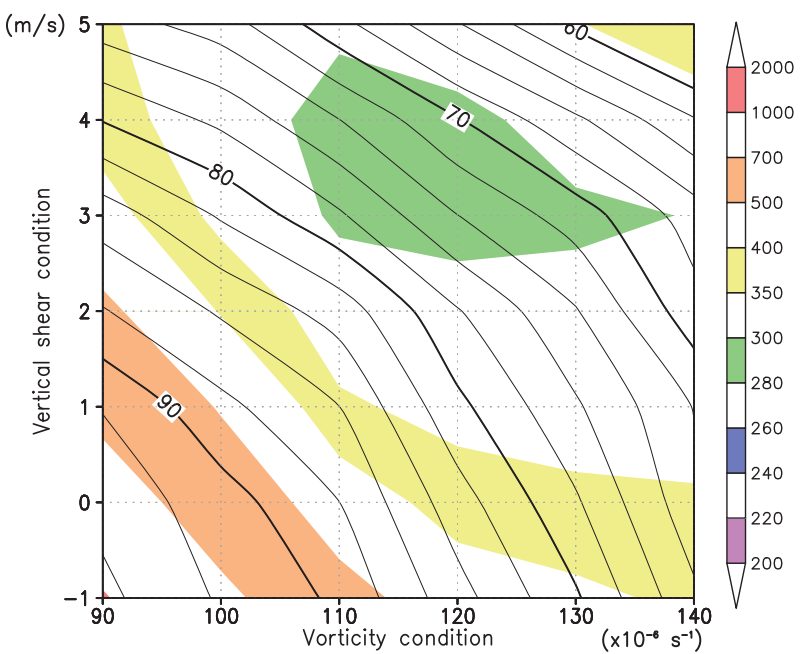

FIG. B1. Hit rate (\%, contour) and total error amount (shade), defined as the sum of misses and false alarms, in the two-dimensional plane spanned by two parameters - the vorticity condition in the horizontal direction and the vertical-shear condition in the vertical direction-the tropical cyclone (TC) tracking by the NEAT algorithm without the warm-core condition. The contour interval is $2 \%$, and the shading is per the legend on the right.

the warm-core condition by tuning the vertical-shear condition $V$, which is the difference between maximum $850-\mathrm{hPa}$ horizontal wind and maximum $300-\mathrm{hPa}$ horizontal wind inside of the TC. We swept it from -1 to $5 \mathrm{~m} \mathrm{~s}^{-1}$. When the value becomes greater, the condition becomes stricter; then, the hit rate must decrease and total error must increase. Figure B1 shows the hit rate and total error in the space of the vorticity versus vertical-shear conditions. The total error attains a local minimum at $(C, V)=\left(120 \times 10^{-6}, 3 \mathrm{~m} \mathrm{~s}^{-1}\right)$, but it is much greater than that for the optimal parameter set provided in section 4 . This parameter yields a much lower hit rate of $\sim 75 \%$. This result suggests that both the vertical-shear and warm-core conditions are necessary in TC identification in this paper.

\section{APPENDIX C}

\section{Failure Cases}

Appendix $\mathrm{C}$ shows the error distribution of TCs for the NEAT and NPT algorithms. The climatological TC track density for the misses (Figs. C1a,c) is uniformly scattered over the typical typhoon path, and the TC track density for the misses is rather low because of the highly tuned algorithm. In contrast, the climatological TC track density for false alarms (Figs. C1b,d) is far northeastward of Japan. These TCs are regarded as extratropical cyclones, which cannot be completely removed in the TC identification process by either method. We also found 
(a) Density distribution of missed TCs (NEAT)

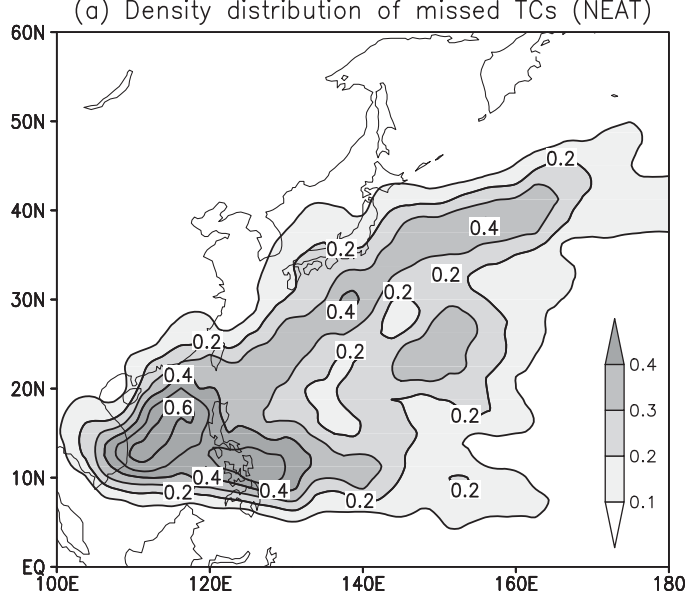

(c) Density distribution of missed TCs (NPT)

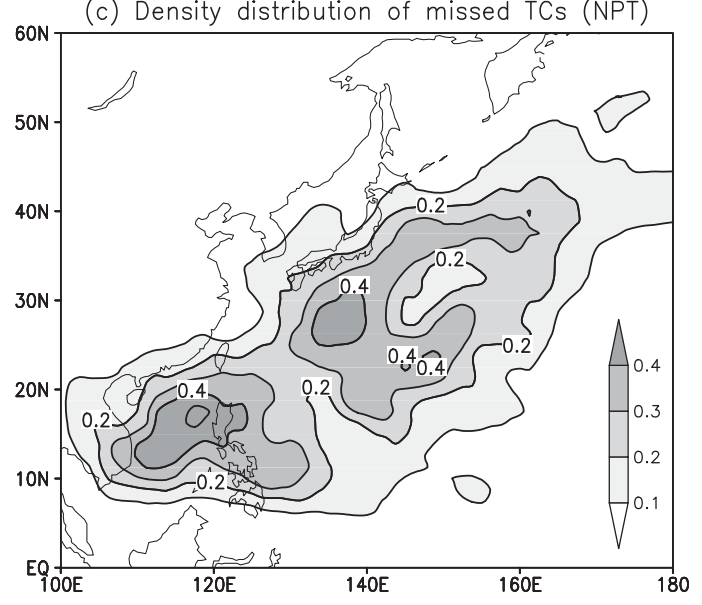

(b) Density distribution of false alarms (NEAT)

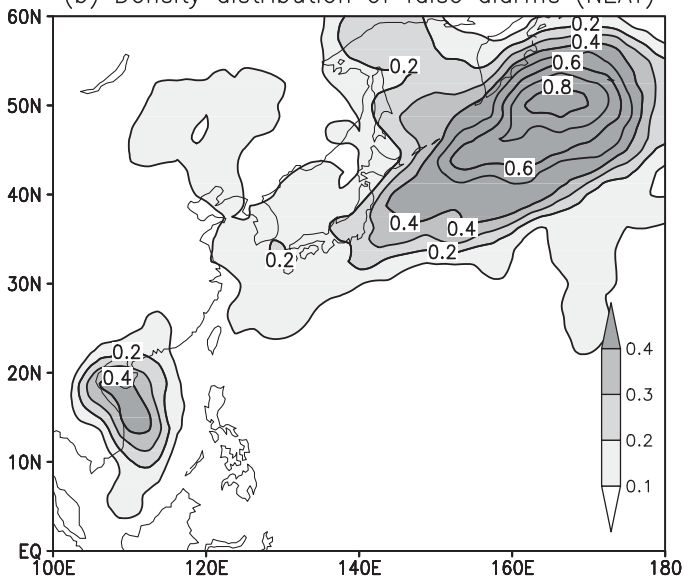

(d) Density distribution of false alarms (NPT)

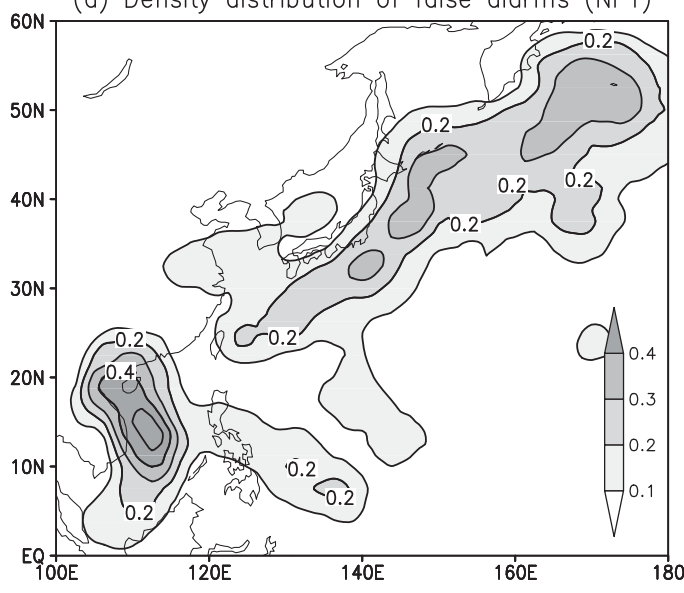

FIG. C1. Climatological annual-mean track density for (a) missed tracks and (b) false-alarm tracks in the NEAT algorithm. The contour interval is $0.1 \mathrm{yr}^{-1}$, and the shading is per the legend on the bottom right. (c),(d) As in (a),(b), but for the NPT algorithm.

a number of false-alarm tracks near the Indochina Peninsula, which are perhaps tropical low pressure systems that do not satisfy the JMA typhoon conditions.

\section{REFERENCES}

Arnaud, Y., M. Desbois, and J. Maizi, 1992: Automatic tracking and characterization of African convective systems on Meteosat pictures. J. Appl. Meteor., 31, 443-453.

Bell, G. D., and Coauthors, 2000: Climate assessment for 1999. Bull. Amer. Meteor. Soc., 81, S1-S50.

Bengtsson, L., M. Botzet, and M. Esch, 1995: Hurricane-type vortices in a general circulation model. Tellus, 47A, 175196.

_ - K. I. Hodges, and M. Esch, 2007: Tropical cyclones in a T159 resolution global climate model: Comparison with observations and reanalysis. Tellus, 59A, 396-416.

Brand, S., 1972: Very large and very small typhoons of the western North Pacific. J. Meteor. Soc. Japan, 50, 332-341.

Broccoli, A. J., and S. Manabe, 1990: Can existing climate models be used to study anthropogenic changes in tropical cyclone climate? Geophys. Res. Lett., 17, 1917-1920.
Chan, J. C. L., and C. K. M. Yip, 2003: Interannual variations of tropical cyclone size over the western North Pacific. Geophys. Res. Lett., 30, 2267, doi:10.1029/2003GL018522.

__ and K. S. Liu, 2004: Global warming and western North Pacific typhoon activity from an observational perspective. J. Climate, 17, 4590-4602.

Denman, S., V. Chandran, and S. Sridharan, 2007: An adaptive optical flow technique for person tracking systems. Pattern Recognit. Lett., 28, 1232-1239.

Emanuel, K., 2005: Increasing destructiveness of tropical cyclones over the past 30 years. Nature, 436, 686-688.

Evans, J. L., and R. E. Hart, 2003: Objective indicators of the onset and completion of ET for Atlantic tropical cyclones: Factors determining post-transition evolution. Mon. Wea. Rev., 131, 909-925.

Haarsma, R. J., F. F. B. Mitchell, and C. A. Senior, 1993: Tropical disturbances in a GCM. Climate Dyn., 8, 247-257.

Hart, R. E., 2003: A cyclone phase space derived from thermal wind and thermal asymmetry. Mon. Wea. Rev., 131, 585-616.

_ 2011: An inverse relationship between aggregate Northern Hemisphere tropical cyclone activity and subsequent winter climate. Geophys. Res. Lett., 38, L01705, doi:10.1029/ 2010GL045612. 
Hasegawa, A., and S. Emori, 2005: Tropical cyclones and associated precipitation over the western North Pacific: T106 atmospheric GCM simulation for present-day and doubled $\mathrm{CO}_{2}$ climates. SOLA, 1, 145-148.

Hatsushika, H., J. Tsutsui, M. Fiorino, and K. Onogi, 2006: Impact of wind profile retrievals on the analysis of tropical cyclones in the JRA-25 reanalysis. J. Meteor. Soc. Japan, 84, 891-905.

Hodges, K. I., 1994: A general method for tracking analysis and its application to meterological data. Mon. Wea. Rev., 122, 2573 2586.

_ 1996: Spherical nonparametric estimators applied to the UGAMP model integration for AMIP. Mon. Wea. Rev., 124, 2914-2932.

Inatsu, M., 2009: The neighbor enclosed area tracking algorithm for extratropical wintertime cyclones. Atmos. Sci. Lett., 10, 267-272.

—_, and S. Amada, 2013: Dynamics and geometry of extratropical cyclones in the upper troposphere by a neighbor enclosed area tracking algorithm. J. Climate, in press.

Jones, S. C., and Coauthors, 2003: The extratropical transition of tropical cyclones: Forecast challenge, current understanding, and future directions. Wea. Forecasting, 18, 1052-1092.

Kimball, S. K., and M. S. Mulekar, 2004: A 15-year climatology of North Atlantic tropical cyclones. Part I: Size parameters. J. Climate, 17, 3555-3575.

Kitabatake, N., 2011: Climatology of extratropical transition of tropical cyclones in the western North Pacific defined by using cyclone phase space. J. Meteor. Soc. Japan, 89, 309-325.

Knutson, T. R., J. J. Sirutis, S. T. Garner, I. M. Held, and R. R. Tuleya, 2007: Simulation of the recent multidecadal increase of Atlantic hurricane activity using an 18-km-grid regional model. Bull. Amer. Meteor. Soc., 88, 1549-1565.

Lee, C.-S., K. K. W. Cheung, W.-T. Fang, and R. L. Elsberry, 2010: Initial maintenance of tropical cyclone size in the western North Pacific. Mon. Wea. Rev., 138, 3207-3223.

Liu, K. S., and J. C. L. Chan, 1999: Size of tropical cyclones as inferred from ERS-1 and ERS-2 data. Mon. Wea. Rev., 127, 2992-3001.

Manganello, J. V., and Coauthors, 2012: Tropical cyclone climatology in a 10-km global atmospheric GCM: Toward weatherresolving climate modeling. J. Climate, 25, 3867-3893.

Mathon, V., and H. Laurent, 2001: Life cycle of Sahelian mesoscale convective cloud systems. Quart. J. Roy. Meteor. Soc., 127, 377-406.

McDonald, R. E., D. G. Bleaken, D. R. Cresswell, V. D. Pope, and C. A. Senior, 2005: Tropical storms: Representation and di- agnosis in climate models and the impacts of climate change. Climate Dyn., 25, 19-36.

Merrill, R. T., 1984: A comparison of large and small tropical cyclones. Mon. Wea. Rev., 112, 1408-1418.

Morel, C., and S. Senesi, 2002: A climatology of mesoscale convective systems over Europe using satellite infrared imagery. I: Methodology. Quart. J. Roy. Meteor. Soc., 128, 1953-1971.

Murakami, H., and B. Wang, 2010: Future change of North Atlantic tropical cyclone tracks. J. Climate, 23, 2699-2721.

Onogi, K., and Coauthors, 2007: The JRA-25 reanalysis. J. Meteor. Soc. Japan, 85, 369-432.

Oouchi, K., J. Yoshimura, H. Yoshimura, R. Mizuta, S. Kusunoki, and A. Noda, 2006: Tropical cyclone climatology in a globalwarming climate as simulated in a $20 \mathrm{~km}$-mesh global atmospheric model: Frequency and wind intensity analysis. J. Meteor. Soc. Japan, 84, 259-276.

Ooyama, K., 1969: Numerical simulation of the life cycle of tropical cyclones. J. Atmos. Sci., 26, 3-40.

- 1982: Conceptual evolution of the theory and modeling of the tropical cyclone. J. Meteor. Soc. Japan, 60, 369-379.

Rudeva, I., and S. K. Gulev, 2007: Climatology of cyclone size characteristics and their changes during the cyclone life cycle. Mon. Wea. Rev., 135, 2568-2587.

Sakamoto, T. T., and Coauthors, 2012: MIROC4-A new highresolution CCSR/NIES/JAMSTEC atmosphere-ocean coupled GCM. J. Meteor. Soc. Japan, 90, 325-359.

Sinclair, M. R., 1997: Objective identification of cyclones and their circulation intensity, and climatology. Wea. Forecasting, 12, 595-612.

Sugi, M., A. Noda, and N. Sato, 2002: Influence of the global warming on tropical cyclone climatology: An experiment with the JMA global model. J. Meteor. Soc. Japan, 80, 249-272.

Ulbrich, U., G. C. Leckebusch, and J. G. Pinto, 2009: Extra-tropical cyclones in the present and future climate: A review. Theor. Appl. Climatol., 96, 117-131.

Walsh, K., M. Fiorino, C. Landsea, and K. McInnes, 2007: Objectively determined resolution-dependent threshold criteria for the detection of tropical cyclones in climate models and reanalyses. J. Climate, 20, 2307-2314.

Wang, B., and J. C. L. Chan, 2002: How strong ENSO events affect tropical storm activity over the western North Pacific. J. Climate, 15, 1643-1658.

Williams, M., and R. A. Houze Jr., 1987: Satellite-observed characteristics of winter monsoon cloud clusters. Mon. Wea. Rev., 115, 505-519. 